\title{
GLOBAL ANALYSIS IN A PREDATOR-PREY SYSTEM WITH NONMONOTONIC FUNCTIONAL RESPONSE*
}

\author{
SHIGUI RUAN ${ }^{\dagger}$ AND DONGMEI XIAO $\ddagger$
}

\begin{abstract}
A predator-prey system with nonmonotonic functional response is considered. Global qualitative and bifurcation analyses are combined to determine the global dynamics of the model. The bifurcation analysis of the model depending on all parameters indicates that it exhibits numerous kinds of bifurcation phenomena, including the saddle-node bifurcation, the supercritical and subcritical Hopf bifurcations, and the homoclinic bifurcation. It is shown that there are different parameter values for which the model has a limit cycle or a homoclinic loop, or exhibits the so-called paradox of enrichment phenomenon. Moreover, a limit cycle cannot coexist with a homoclinic loop for all parameters. In the generic case, the model has the bifurcation of cusp type of codimension 2 (i.e., Bogdanov-Takens bifurcation) but for some specific parameter values it has a multiple focus of multiplicity at least 2 .
\end{abstract}

Key words. predator-prey system, global analysis, Bogdanov-Takens bifurcation, homoclinic orbit, Hopf bifurcation, limit cycle, paradox of enrichment

AMS subject classifications. Primary, 34C25, 92D25; Secondary, 58F14

PII. S0036139999361896

1. Introduction. In population dynamics, a functional response of the predator to the prey density refers to the change in the density of prey attached per unit time per predator as the prey density changes. In microbial dynamics or chemical kinetics, the functional response describes the uptake of substrate by the microorganisms. The simplest model of functional response is obtained by assuming that in the time available for searching, the total change in the prey density/substrate concentration is proportional to the prey density/substrate concentration. Hence, if $x(t)$ represents the prey density/substrate concentration at time $t$, then the functional response is $a x(t)$, where $a>0$ is a constant. Such a response was used almost simultaneously by Lotka in 1925 in studying a hypothetical chemical reaction and by Volterra in 1926 in modeling a predator-prey interaction. However, the curve defined by the LotkaVolterra response function is a straight line through the origin and is unbounded. Thus, more reasonable response functions should be nonlinear and bounded. In 1913, Michaelis and Menten proposed the response function

$$
p(x)=\frac{m x}{a+x}
$$

in studying enzymatic reactions, where $m>0$ denotes the maximal growth rate of the species and $a>0$ is the half-saturation constant. In 1959, Holling [20] also used this function as one of the predator functional responses. It is now referred to as a Michaelis-Menten function or a Holling type-II function. Another class of response

* Received by the editors October 21, 1999; accepted for publication (in revised form) August 25, 2000; published electronically January 16, 2001.

http://www.siam.org/journals/siap/61-4/36189.html

${ }^{\dagger}$ Department of Mathematics and Statistics, Dalhousie University, Halifax, Nova Scotia, Canada B3H 3J5 (ruan@mscs.dal.ca). The research of this author was supported by the Natural Science and Engineering Research Council of Canada.

${ }^{\ddagger}$ Department of Mathematics, Central China Normal University, Wuhan, Hubei 430079, China (dmxiao@ccnu-1.ccnu.edu.cn). The research of this author was supported by the National Natural Science Foundations of China. 
function is

$$
p(x)=\frac{m x^{2}}{a+b x+x^{2}},
$$

which is called a sigmoidal response function, while the simplification

$$
p(x)=\frac{m x^{2}}{a+x^{2}}
$$

is known as a Holling type-III function. For some other types of response functions, we refer to Freedman [13]. In general the response function $p(x)$ satisfies the following general hypothesis (see Hale and Somolinos [19]):

(A) $p(x)$ is a continuously differentiable function defined on $[0, \infty)$ and satisfies

$$
p(0)=0, \quad p^{\prime}(x)>0, \text { and } \lim _{x \rightarrow \infty} p(x)=m<\infty .
$$

The inherent assumption in $(\mathrm{A})$ is that $p(x)$ is monotonic, which is true in many predator-prey interactions (see, e.g., Freedman [13], Hsu [22], Kooij and Zegeling [23], Kuang and Freedman [25], May [27], Sugie, Kohno, and Miyazaki [32], and the references cited therein). However, there is experimental and observational evidence that indicates that this need not always be the case, for example, in the cases of "inhibition" in microbial dynamics and "group defense" in population dynamics.

There are experiments (Andrews [1], Boon and Landelout [6], Edwards [12], Yang and Humphrey [39], etc.) that indicate that nonmonotonic responses occur at the microbial level: when the nutrient concentration reaches a high level an inhibitory effect on the specific growth rate may occur. This is often seen when microorganisms are used for waste decomposition or for water purification. To model such an inhibitory effect, Andrews [1] suggested a function

$$
p(x)=\frac{m x}{a+b x+x^{2}},
$$

called the Monod-Haldane function, which is similar to the Monod (i.e., the MichaelisMenten) function for low concentrations but includes the inhibitory effect at high concentrations. Collings [9] also used the response function (1.5) in a mite predatorprey interaction model and called it a Holling type-IV function (see Taylor [34]). The experiments of Edwards [12] supported the use of the function (1.5) for the dependence of the growth rate on an inhibitory substrate. In experiments on the uptake of phenol by pure culture of Pseudomonas putida growing on phenol in continuous culture, Sokol and Howell [31] proposed a simplified Monod-Haldane function of the form

$$
p(x)=\frac{m x}{a+x^{2}}
$$

and found that it fits their experimental data significantly better and is simpler since it involves only two parameters. We can see that if the Monod-Haldane or Holling typeIV function (1.5) is regarded as a modification of the sigmoidal response function (1.2), then the simplified Monod-Haldane or Holling type-IV function (1.6) is a modification of the Holling type-III function (1.3).

In population dynamics, group defense is a term used to describe the phenomenon whereby predation is decreased, or even prevented altogether, due to the increased ability of the prey to better defend or disguise themselves when their numbers are 


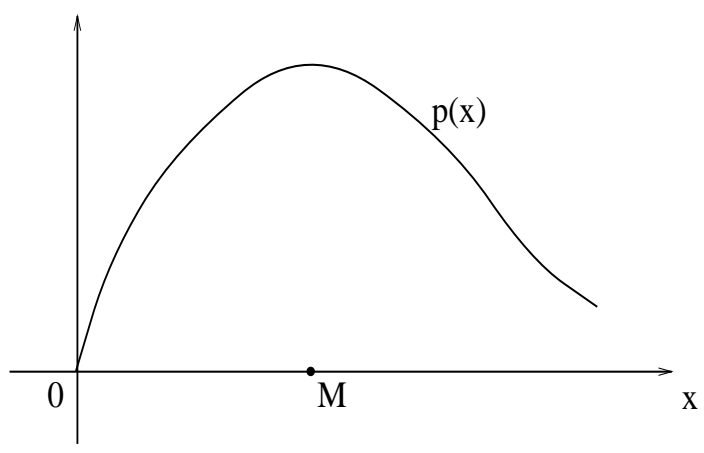

FIG. 1.1. The nonmonotonic predator response function $p(x)$.

large enough. An example of this phenomenon is described by Tener [35]. Lone musk ox can be successfully attacked by wolves. Small herds of musk ox (2 to 6 animals) are attacked but with rare success. No successful attacks have been observed in larger herds. A second example described by Holmes and Bethel [21] involves certain insect populations. Apparently, large swarms of insects make individual identification difficult for their predators. The third example was observed by Davidowicz, Gliwicz, and Gulati [10]. Filamenteous algae are often qualified as inedible by herbivorous zooplankton. However, experiments show that Daphnia can consume them at low concentrations, while they jam their filtering apparatus in high concentrations.

To study the predator-prey interaction when the prey exhibits group defense, Freedman and Wolkowicz [14], Mischaikow and Wolkowicz [28], and Wolkowicz [36] proposed the following model (see also Lin [26]):

$$
\begin{aligned}
& \dot{x}=x g(x, K)-y p(x), \\
& \dot{y}=y(-D+q(x)) .
\end{aligned}
$$

Here, $x$ and $y$ are functions of time representing population densities of prey and predator, respectively; $K>0$ is the carrying capacity of the prey and $D>0$ is the death rate of the predator. The function $g(x, K)$ represents the specific growth rate of the prey in the absence of predator and is assumed to satisfy the following conditions (for $x \geq 0, K>0$ ):

$$
\begin{aligned}
& g(K, K)=0, \quad g(0, K)>0, \quad \lim _{K \rightarrow \infty} g(0, K)<\infty, \\
& g_{x}(x, K)<0, \quad g_{K}(x, K) \geq 0, \quad g_{x K}(x, K)>0, \quad \lim _{K \rightarrow \infty} g_{x}(x, K)=0 .
\end{aligned}
$$

A prototype is the logistic growth

$$
g(x, K)=r\left(1-\frac{x}{K}\right),
$$

which satisfies all the conditions. The function $p(x)$ denotes the predator response function and it is assumed that $p(x)$ satisfies

$$
p(0)=0, p(x)>0 \text { for } x>0 .
$$

In order to model group defense, it is assumed that there is a constant $M>0$ such that (see Figure 1.1)

$$
p^{\prime}(x) \begin{cases}>0, & 0 \leq x<M \\ <0, & x>M .\end{cases}
$$



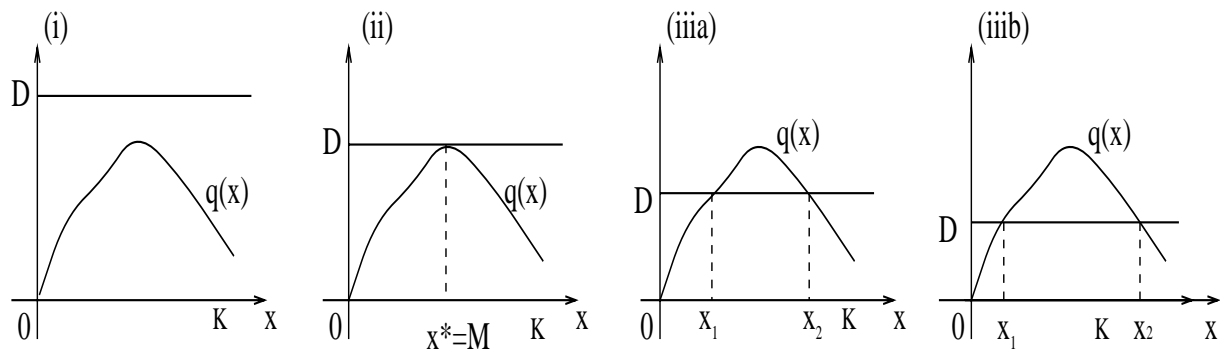

FIG. 1.2. (i) $q(M)<D$; (ii) $q(M)=D$; (iiia)-(iiib) $q(M)>D$.

In particular, the Monod-Haldane or Holling type-IV function (1.5), the simplified version (1.6), and the function $p(x)=\alpha x e^{-\beta x}$ satisfy the assumptions; $\alpha$ and $\beta$ are positive constants. The rate of conversion of prey to predator is described by $q(x)$. In Gause's model, $q(x)=c p(x)$ for some positive constant $c$. It is assumed that $q(x)$ has properties similar to $p(x)$, that is,

$$
q(0)=0, q(x)>0 \text { for } x>0
$$

and

$$
q^{\prime}(x) \begin{cases}>0, & 0 \leq x<M \\ <0, & x>M\end{cases}
$$

The existence of $M>0$ is precisely the assumption which models group defense. It is assumed that the same $M$ holds for both functions $p$ and $q$ since the conversation of prey to predator should increase and decrease as the consumption of prey increases and decreases.

Freedman and Wolkowicz [14] showed that if the carrying capacity of the prey population is sufficiently large, the predator population is almost always driven to extinction. This is related to the phenomenon of paradox of enrichment as described in Rosenzweig [29]. Wolkowicz [36] showed that if the carrying capacity is made sufficiently large by enrichment of the environment, the model predicts the eventual extinction of the predator. In fact, she showed that there is actually a separatrix: for all solutions initiating on one side of the separatrix, extinction of the predator results; solutions initiating on the other side of the separatrix converge to an interior equilibrium. The $x$-component of any interior equilibrium of system (1.7) satisfies the algebraic equation

$$
q(x)=D .
$$

There are three possibilities (see Figure 1.2): (i) $q(M)<D$. In this case, system (1.7) has no interior equilibrium. (ii) $q(M)=D$. In this case, system (1.7) has a unique interior equilibrium, denoted by $E^{*}=\left(x^{*}, y^{*}\right)$ if $x^{*}<K$. (iii) $q(M)>D$. In this case, system (1.7) has two interior equilibria, $E_{1}=\left(x_{1}, y_{1}\right)$ and $E_{2}=\left(x_{2}, y_{2}\right)$, provided $x_{1}<x_{2}<K$ (see Figure 1.2(iiia)). And system (1.7) has a unique interior equilibrium $E_{1}=\left(x_{1}, y_{1}\right)$ if $x_{1}<K<x_{2}$ (see Figure 1.2(iiib)).

Case (i) is not interesting. In case (iiia), according to Wolkowicz [36], $E_{1}$ is stable (a node or focus) if

$$
\frac{\partial}{\partial x}\left(\frac{x g(x, K)}{p(x)}\right)<0
$$



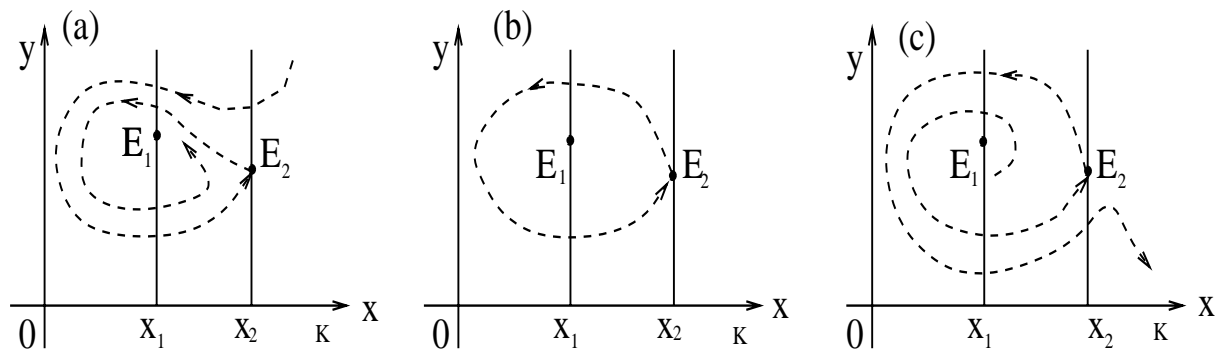

FIG. 1.3. Three possibilities of the portion of the stable and unstable manifold of $E_{2}$ to the left of $x_{2}$.

and $E_{2}=\left(x_{2}, y_{2}\right)$ is unstable (a saddle). By regarding $K$, the carrying capacity of the prey, as a bifurcation parameter, Wolkowicz [36] showed that the system undergoes a Hopf bifurcation at $E_{1}$ when $K$ passes through a critical value. With the presence of the two interior equilibria, Freedman and Wolkowicz [14] observed that there are three possibilities (see Figure 1.3).

In Figure 1.3(b), we can see that there is a homoclinic orbit surrounding $E_{1}$. Recall that a periodic orbit bifurcates from $E_{1}$ when $K$ passes through some critical value. Their numerical analysis indicated that the periodic orbit coalesces with the homoclinic orbit when $K$ varies. Based on this observation, Wolkowicz [36] then argued that there exists a homoclinic bifurcation associated with the Hopf bifurcation (either the Hopf bifurcation followed by the homoclinic bifurcation or the homoclinic bifurcation followed by the Hopf bifurcation) when the same bifurcation parameter $K$ takes some other value. The observation and analysis by Freedman and Wolkowicz [14] and Wolkowicz [36] show that system (1.7) has very interesting and rich dynamics. Let us reconsider the existence of the interior equilibria. In case (i) there is no interior equilibrium, in case (ii) there is only one, and in case (iii) there are at most two (a saddle and a node (or focus)). It follows from case (i) to case (iii) that system (1.7) exhibits a saddle-node bifurcation. This bifurcation occurs when either $D$ or a parameter involved in $p(x)$ varies. Freedman and Wolkowicz [14] and Wolkowicz [36] studied one situation in case (iii) when system (1.7) has two interior equilibria $E_{1}=\left(x_{1}, y_{1}\right)$ and $E_{2}=\left(x_{2}, y_{2}\right)$ with $x_{1}<x_{2}<K$ and only regarded $K$ as a bifurcation parameter, while case (ii) has never been investigated in the literature. Then it is very natural to ask the following questions:

(1) If there is a unique interior equilibrium, what is its property?

(2) How many kinds of bifurcations can the model exhibit?

(3) What are the global dynamics of the model depending on all parameters?

To have a clear and global bifurcation picture and corresponding phase pictures about system (1.7), we consider $g(x, K)$ given by the logistic function, $p(x)$ the simplified Monod-Haldane or Holling type-IV function given by (1.6), and $q(x)=c p(x)$. Scaling the parameter $m$ (and then changing $r$, but we still denote it by $r$ ) in the first equation and writing $\mu=m c$ in the second equation, we have the following system:

$$
\begin{aligned}
& \dot{x}=r x\left(1-\frac{x}{K}\right)-\frac{x y}{a+x^{2}}, \\
& \dot{y}=y\left(\frac{\mu x}{a+x^{2}}-D\right) .
\end{aligned}
$$

From the point of view of biology, we only restrict our attention to system (1.9) in the closed first quadrant in the $(x, y)$ plane. 
By studying question (1), we can clearly observe the so-called paradox of enrichment phenomenon depending on the dynamics of the system. Now let us consider question (2). System (1.9) has five positive parameters, $r, K, a, \mu$, and $D$. When these parameters change, system (1.9) has a sequence of bifurcations such as a saddlenode bifurcation, a supercritical Hopf bifurcation, a subcritical Hopf bifurcation, a homoclinic bifurcation, etc. By the results in Guckenheimer [15], we know that these bifurcations cannot be obtained by varying just one parameter. From analysis, we shall see that the parameter $r$ cannot be a bifurcation parameter; however, the other parameters can become bifurcation parameters. As discussed above, the saddle-node bifurcation depends on one parameter; the Hopf bifurcation studied in Wolkowicz [36] in fact depends on another parameter. However, the homoclinic bifurcation (i.e., the brokenness of the homoclinic orbit) may depend on both parameters. Therefore, the codimension of these bifurcations must be at least 2 (see also Chow, $\mathrm{Li}$, and Wang [8], Guckenheimer and Holmes [16], and Kuznetsov [24]). Even though we can prove that the unique degenerate interior equilibrium $E^{*}$ is a cusp of codimension 2 and the system exhibits a Bogdanov-Takens bifurcation (see Bogdanov $[4,5]$ and Takens [33]), it is possible that system (1.9) has more than two limit cycles since there exists a multiple focus of multiplicity at least 2 . Therefore, the dynamics of system (1.9) could be much more complicated than we may have expected.

We shall make global qualitative and bifurcation analyses of system (1.9) depending on all parameters and show that system (1.9) has a unique limit cycle or a global attractive equilibrium in the interior of the first quadrant, or exhibits the paradox of enrichment phenomenon for different parameter values. However, it cannot have a limit cycle and a homoclinic loop simultaneously for all parameters. Furthermore, we show that system (1.9) can exhibit qualitatively different dynamics, including the supercritical and subcritical Hopf bifurcations, the saddle-node bifurcation as well as the homoclinic bifurcation. In the generic case, system (1.9) has a bifurcation of codimension 2 (i.e., the Bogdanov-Takens bifurcation) and no bifurcations of codimension 3. We also present a global bifurcation picture near the cusp. The global bifurcation diagram enables us to link these bifurcations one to another by using the two bifurcation parameters, namely, $K$ (the carrying capacity of the prey) and $D$ (the death rate of the predator).

We would like to mention that one could use the Bogdanov-Takens bifurcation theorem stated in Kuznetsov [24] to show that system (1.9) exhibits a BogdanovTakens bifurcation. However, to find the global bifurcation diagram and to locate the bifurcation curves in terms of the parameters in system (1.9), one still has to calculate the unfoldings. Instead of applying the Bogdanov-Takens bifurcation theorem in Kuznetsov [24] directly, we use a series of (nontrivial) transformations to compute the normal form, and thus we are able to determine the bifurcation curves and the diagram by using the original parameters in system (1.9).

This paper is organized as follows. General phase portrait analysis of the system (1.9) is carried out in section 2. We study all possible bifurcations depending on all parameters and describe the phase portraits and the biological ramifications. Moreover, we show that the system has a unique limit cycle for some parameters, has a global attractive equilibrium in the interior of the first quadrant for some other parameters, and cannot have both a limit cycle and a homoclinic loop for all parameters. In section 3 we consider a degenerate equilibrium. We show that in a small neighborhood of the degenerate equilibrium, system (1.9) undergoes the Bogdanov-Takens bifurcation in the generic case, i.e., the bifurcation of the cusp type of codimension 
2 and no bifurcation of codimension 3 when the parameters vary in a small neighborhood of some parameter values. Finally, we choose two parameters, $K$ and $D$, as the bifurcation parameters and show that system (1.9) exhibits the Bogdanov-Takens bifurcation. For different parameters, the system could have a unique limit cycle or a homoclinic loop. The paper ends with a brief discussion.

2. General phase portraits analysis of the system (1.9). As is typical for predator-prey systems, the $x$-axis, $y$-axis, and interior of the first quadrant are all invariant under system (1.9); solutions with positive initial values are positive and bounded; and there is a hyperbolic saddle point at the origin and an equilibrium $(K, 0)$ in the $x$-axis for all permissible parameters. The equilibrium of the greatest interest would be an equilibrium interior to the first quadrant, so we seek conditions for such an equilibrium to exist. From system (1.9), we can see that if there is an interior equilibrium, then the equation

$$
\frac{\mu x}{a+x^{2}}-D=0
$$

that is,

$$
D x^{2}-\mu x+a D=0,
$$

has positive roots. Therefore, the first condition is that

$$
\mu^{2}-4 a D^{2} \geq 0
$$

Biologically, this is necessary for the persistence of the ecosystem, for if $\mu^{2}-4 a D^{2}<0$, then $\frac{\mu x}{a+x^{2}}-D<0$ for all $x>0$ and $\dot{y}(t)<0$. Hence $\lim _{t \rightarrow \infty} y\left(t, t_{0}, x_{0}, y_{0}\right)=0$ as $x_{0}>0, y_{0}>0$. This implies that the predator species goes extinct. Mathematically, $\mu^{2}-4 a D^{2}=0$ is a saddle-node bifurcation surface. When the parameters pass from one side of the surface to the other side, the number of equilibria of the system changes. To determine the $y$ value of the equilibrium, we merely solve

$$
r x\left(1-\frac{x}{K}\right)-y \frac{x}{a+x^{2}}=0
$$

for $y$ at the root $x^{*}$ of $(2.1)$ and get

$$
y^{*}=r\left(1-\frac{x^{*}}{K}\right)\left(a+\left(x^{*}\right)^{2}\right) .
$$

To ensure that $y^{*}>0$, however, we must make a second assumption:

$$
x^{*}<K .
$$

Notice that if the predator response function is monotone, Freedman [13] has shown that if $x^{*}>K$, then the predator goes extinct. However, if the predator response function is nonmonotone, as in system (1.7), there could be two roots $x_{1}$ and $x_{2}$ of (1.8). Even if one $x_{i}>K$, the predator does not necessarily go to extinction. We will see more details in the following.

Next we discuss the possible phase portraits of system (1.9) according to conditions (2.2) and (2.4).

2.1. $\boldsymbol{\mu}^{2}-\mathbf{4 a} \boldsymbol{D}^{2}<\mathbf{0}$. In this case, system (1.9) has no interior equilibria. It is easy to see that the equilibrium $(K, 0)$ is a stable node. 


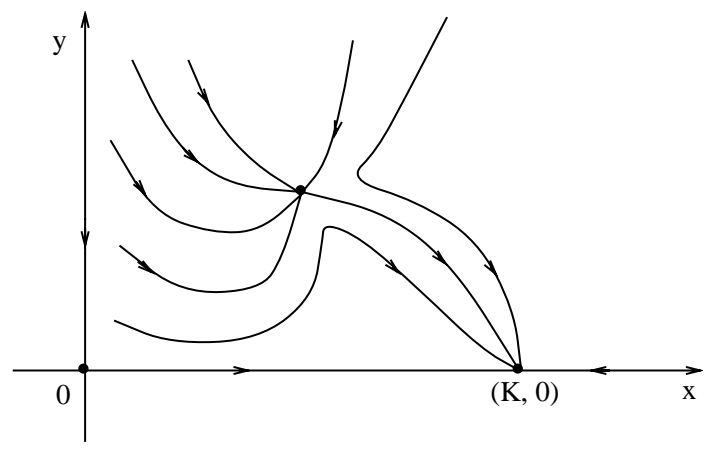

FIG. 2.1. The phase portrait of system (1.9) when $\mu^{2}-4 a D^{2}=0$ but $\frac{\mu}{2 D}<K<\frac{\mu}{D}$.

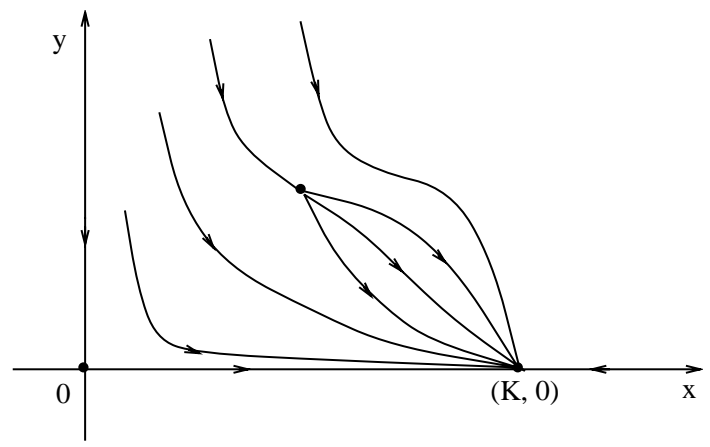

FIG. 2.2. The phase portrait of system (1.9) when $\mu^{2}-4 a D^{2}=0$ but $K>\frac{\mu}{D}$.

2.2. $\boldsymbol{\mu}^{2}-4 a D^{2}=0$ and $\frac{\mu}{2 D}<\boldsymbol{K}$. In this case, system (1.9) has three equilibria, a hyperbolic saddle $(0,0)$, a stable node $(K, 0)$, and an interior equilibrium $\left(x_{0}, y_{0}\right)$, where $x_{0}=\frac{\mu}{2 D}, y_{0}=r\left(1-\frac{x_{0}}{K}\right)\left(a+x_{0}^{2}\right)$. After some calculations, we know that $\left(x_{0}, y_{0}\right)$ is a saddle-node as $\mu \neq K D$. The detailed results will follow.

2.2.1. $\boldsymbol{K}<\frac{\boldsymbol{\mu}}{\boldsymbol{D}}$. The saddle-node $\left(x_{0}, y_{0}\right)$ consists of two hyperbolic sectors and one parabolic sector; the parabolic sector is between the $y$-axis and the equilibrium. The phase portrait can be sketched as in Figure 2.1.

In this case, there exist two seperatrices. Solutions initiating on one side of the seperatrix converge to the interior equilibrium. Thus, the population of the predator and the population of the prey will tend to a steady state if their initial populations lie in the parabolic sector, and the predator will tend to extinction as its initial population density lies in one of the two hyperbolic sectors.

2.2.2. $\boldsymbol{K}>\frac{\mu}{D}$. The saddle-node $\left(x_{0}, y_{0}\right)$ consists of two hyperbolic sectors and one parabolic sector; the hyperbolic sectors lie between the $y$-axis and the equilibrium. The phase portrait is depicted in Figure 2.2.

In this case, there exists one seperatrix which converges to the interior equilibrium, and all other solutions tend to the equilibrium $(K, 0)$. Therefore, sufficient enrichment of the environment leads to extinction of the predator for almost all initial values, which strongly supports the so-called paradox of enrichment.

2.2.3. $\boldsymbol{\mu}=\boldsymbol{K} \boldsymbol{D}$. The equilibrium $\left(x_{0}, y_{0}\right)$ is a cusp which consists of two hyperbolic sectors and two seperatrices. One of the seperatrices converges to the interior 


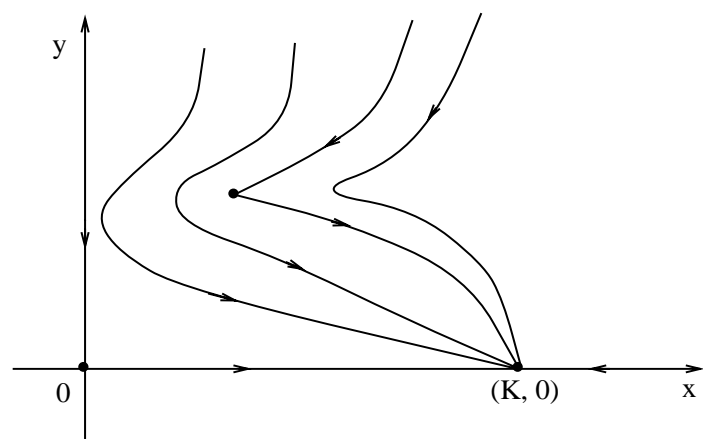

FIG. 2.3. The phase portrait of system (1.9) when $\mu^{2}-4 a D^{2}=0$ but $K=\frac{\mu}{D}$.

equilibrium $\left(x_{0}, y_{0}\right)$ and all other solutions tend to the equilibrium $(K, 0)$. Hence, in this case the paradox of enrichment also holds. The phase portrait is shown in Figure 2.3 .

In section 3, we will prove that the surface

$$
B T: \mu^{2}-4 a D^{2}=0, \quad \mu=K D
$$

is a cusp bifurcation surface of codimension 2 for system (1.9) (i.e., Bogdanov-Takens bifurcation surface).

2.3. $\boldsymbol{\mu}^{\mathbf{2}}-\mathbf{4} \boldsymbol{a} \boldsymbol{D}^{\mathbf{2}}>\mathbf{0}$. Now system (1.9) has at most four equilibria, $(0,0)$, $(K, 0)$, and two interior equilibria $\left(x_{1}, y_{1}\right),\left(x_{2}, y_{2}\right)$, where

$$
\begin{array}{ll}
x_{1}=\frac{\mu-\sqrt{\mu^{2}-4 a D^{2}}}{2 D}, & y_{1}=r\left(1-\frac{x_{1}}{K}\right)\left(a+x_{1}^{2}\right), \\
x_{2}=\frac{\mu+\sqrt{\mu^{2}-4 a D^{2}}}{2 D}, & y_{2}=r\left(1-\frac{x_{2}}{K}\right)\left(a+x_{2}^{2}\right) .
\end{array}
$$

For the sake of simplicity, we also denote

$$
x_{3}=\frac{2 \mu-\sqrt{\mu^{2}-4 a D^{2}}}{2 D}, x_{4}=\frac{2 \mu+\sqrt{\mu^{2}-4 a D^{2}}}{2 D} .
$$

More precisely, there are three possibilities.

(a) When $K \leq x_{1}$, system (1.9) has two boundary equilibria, $(0,0)$ and $(K, 0)$, and no interior equilibria. The dynamics of system (1.9) is trivial.

(b) When $x_{1}<K<x_{2}$, system (1.9) has three equilibria, $(0,0),(K, 0)$, and an interior equilibrium $\left(x_{1}, y_{1}\right)$. In this case the interior equilibrium $\left(x_{1}, y_{1}\right)$ is a focus or node.

(c) When $K>x_{2}$, system (1.9) has four equilibria, a hyperbolic saddle $(0,0)$, a hyperbolic stable node $(K, 0)$, and two interior equilibria $\left(x_{1}, y_{1}\right)$ and $\left(x_{2}, y_{2}\right)$. We can see that $\left(x_{2}, y_{2}\right)$ is a hyperbolic saddle and $\left(x_{1}, y_{1}\right)$ is a focus or node.

Next we discuss the dynamics of system (1.9) in detail.

Lemma 2.1. If $4 a D^{2}<\mu^{2}<\frac{18+2 \sqrt{6}}{3} a D^{2}$ and $K=x_{3}$, then the equilibrium $\left(x_{1}, y_{1}\right)$ of system (1.9) is a stable multiple focus of multiplicity 1 . If $\mu^{2}>\frac{18+2 \sqrt{6}}{3} a D^{2}$ and $K=x_{3}$, then the equilibrium $\left(x_{1}, y_{1}\right)$ of system (1.9) is an unstable multiple focus of multiplicity 1 . And if $\mu^{2}=\frac{18+2 \sqrt{6}}{3} a D^{2}$ and $K=x_{3}$, the equilibrium $\left(x_{1}, y_{1}\right)$ of system (1.9) is a multiple focus of multiplicity at least 2. 
Proof. Let $\bar{x}=x-x_{1}, \bar{y}=y-y_{1}$, and let $d t=\left(a+x^{2}\right) d \tau$. Then when $K=x_{3}$, system (1.9) can be transformed into

$$
\begin{aligned}
& \frac{d \bar{x}}{d \tau}=-x_{1} \bar{y}+r\left(-\frac{a}{K}+3 x_{1}-\frac{6 x_{1}^{2}}{K}\right) \bar{x}^{2}-\bar{x} \bar{y}-r\left(1-\frac{4 x_{1}}{K}\right) \bar{x}^{3}-\frac{r}{K} \bar{x}^{4}, \\
& \frac{d \bar{y}}{d \tau}=\left(\mu-2 D x_{1}\right) y_{1} \bar{x}-D y_{1} \bar{x}^{2}+\left(\mu-2 D x_{1}\right) \bar{x} \bar{y}-D \bar{x}^{2} \bar{y} .
\end{aligned}
$$

According to the formula for the third focal value (i.e., the Liapunov number) of a multiple focus on p. 253 of Andronov [2], we obtain, after elementary but lengthy computations, the following expression for the third focal value $\alpha_{3}$ of the multiple focus $(0,0)$ in system $(2.5)$ :

$$
\begin{aligned}
\alpha_{3} & =\frac{3 \pi x_{1} y_{1} r}{2 K D\left(x_{1} y_{1}\left(\mu-2 D x_{1}\right)\right)^{\frac{3}{2}}}\left(2 \mu x_{1}-10 a D+\frac{\mu^{2}}{D}\right) \\
& =\frac{3 \pi r}{2 K D\left(\mu-2 D x_{1}\right)\left(x_{1} y_{1}\left(\mu-2 D x_{1}\right)\right)^{\frac{1}{2}}}\left(2 \mu^{2}-\mu \sqrt{\mu^{2}-4 a D^{2}}-10 a D^{2}\right) .
\end{aligned}
$$

When $4 a D^{2}<\mu^{2}<\frac{18+2 \sqrt{6}}{3} a D^{2}, \alpha_{3}<0$. Hence, the equilibrium $\left(x_{1}, y_{1}\right)$ is a stable multiple focus of multiplicity 1 . When $\mu^{2}>\frac{18+2 \sqrt{6}}{3} a D^{2}, \alpha_{3}>0$. Then the equilibrium $\left(x_{1}, y_{1}\right)$ is an unstable multiple focus of multiplicity 1 . When $\mu^{2}=$ $\frac{18+2 \sqrt{6}}{3} a D^{2}, \alpha_{3}=0$. Thus, the equilibrium $\left(x_{1}, y_{1}\right)$ is a multiple focus of multiplicity at least 2.

By Lemma 2.1, we know that the equilibrium $\left(x_{1}, y_{1}\right)$ is a stable focus when $4 a D^{2}<\mu^{2}<\frac{18+2 \sqrt{6}}{3} a D^{2}$ and $x_{1}<K \leq x_{3}$. However, the equilibrium $\left(x_{1}, y_{1}\right)$ is an unstable focus as $4 a D^{2}<\mu^{2}<\frac{18+2 \sqrt{6}}{3} a D^{2}$ and $K>x_{3}$.

Hence, when parameters pass from one side of the following surface to the other side, system (1.9) can undergo a supercritical Hopf bifurcation. A stable limit cycle appears in the small neighborhood of $\left(x_{1}, y_{1}\right)$. The surface

$$
H_{1}: \quad K=x_{3}, \quad 4 a D^{2}<\mu^{2}<\frac{18+2 \sqrt{6}}{3} a D^{2}
$$

is called the supercritical Hopf bifurcation surface of system (1.9).

On the other hand, the equilibrium $\left(x_{1}, y_{1}\right)$ is an unstable focus when $\mu^{2}>$ $\frac{18+2 \sqrt{6}}{3} a D^{2}$ and $K \geq x_{3}$; however, it is a stable focus as $\mu^{2}>\frac{18+2 \sqrt{6}}{3} a D^{2}$ and $x_{1}<K<x_{3}$. Therefore, when parameters pass from one side of the following surface to the other side, system (1.9) can undergo a subcritical Hopf bifurcation. An unstable limit cycle appears in the small neighborhood of $\left(x_{1}, y_{1}\right)$. The surface

$$
H_{2}: K=x_{3}, \quad \mu^{2}>\frac{18+2 \sqrt{6}}{3} a D^{2}
$$

is called the subcritical Hopf bifurcation surface of system (1.9).

The surface

$$
H_{0}: \quad K=x_{3}, \quad \mu^{2}=\frac{18+2 \sqrt{6}}{3} a D^{2}
$$

is called the degenerate Hopf bifurcation surface of system (1.9). 


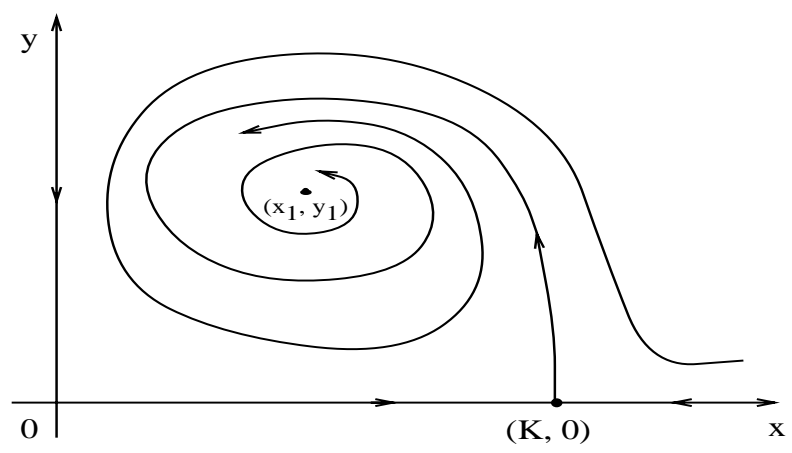

FIG. 2.4. The phase portrait of system (1.9) when $4 a D^{2}<\mu^{2} \leq \frac{16}{3} a D^{2}$ and $x_{1}<K<x_{2}$.

Theorem 2.2. If $4 a D^{2}<\mu^{2} \leq \frac{16}{3} a D^{2}$ and $x_{1}<K<x_{2}$, then system (1.9) has three equilibria: two hyperbolic saddles $(0,0)$ and $(K, 0)$ and a globally asymptotically stable equilibrium $\left(x_{1}, y_{1}\right)$ in the interior of the first quadrant. The phase portrait is shown in Figure 2.4.

Proof. It is easily to check that the equilibrium $\left(x_{1}, y_{1}\right)$ is a stable focus (or node). Next we prove that system (1.9) has no periodic orbits in the interior of the first quadrant.

It is clear that the dynamics of system (1.9) are equivalent to that of the system

$$
\begin{aligned}
& \frac{d x}{d \tau}=x\left(r\left(1-\frac{x}{K}\right)\left(a+x^{2}\right)-y\right), \\
& \frac{d y}{d \tau}=y\left(\mu x-D\left(a+x^{2}\right)\right),
\end{aligned}
$$

where $d t=\left(a+x^{2}\right) d \tau$.

Taking the Dulac function $D(x, y)=x^{-1} y^{-1}$ for system (2.6) and noting that $K \leq \sqrt{3 a}$ if $4 a D^{2}<\mu^{2} \leq \frac{16}{3} a D^{2}$ and $x_{1}<K<x_{2}$, we can see that system (2.6) has no periodic orbits in the interior of the first quadrant. Hence the equilibrium $\left(x_{1}, y_{1}\right)$ is globally asymptotically stable in the interior of the first quadrant.

Remark 2.3. In Theorem 2.2 if $K=x_{2}$, then the equilibrium $\left(x_{2}, y_{2}\right)$ goes to the equilibrium $(K, 0)$ such that the equilibrium $(K, 0)$ is a complex equilibrium. By analysis we readily find the equilibrium $(K, 0)$ is a saddle-node equilibrium and $\left(x_{1}, y_{1}\right)$ is a globally asymptotically stable equilibrium in the interior of the first quadrant.

THEOREM 2.4. If $\mu^{2}>\frac{16}{3} a D^{2}$ and $x_{2}>K>x_{3}$, then system (1.9) has at least one limit cycle in the interior of the first quadrant.

Proof. It is clear that the periodic orbit of system (1.9) must be in the domain $E_{1}$ if it exists, where

$$
E_{1}=\{(x, y): 0<x<K, 0<y<+\infty\} .
$$

Taking a ray line $l_{1}$ beginning at the point $A(K, 0)$

$$
l_{1}=\{(x, y): x=K, y>0\},
$$

we can see that the direction vector field of system (1.9) on $l_{1}$ is from right to left (see Figure 2.5). 


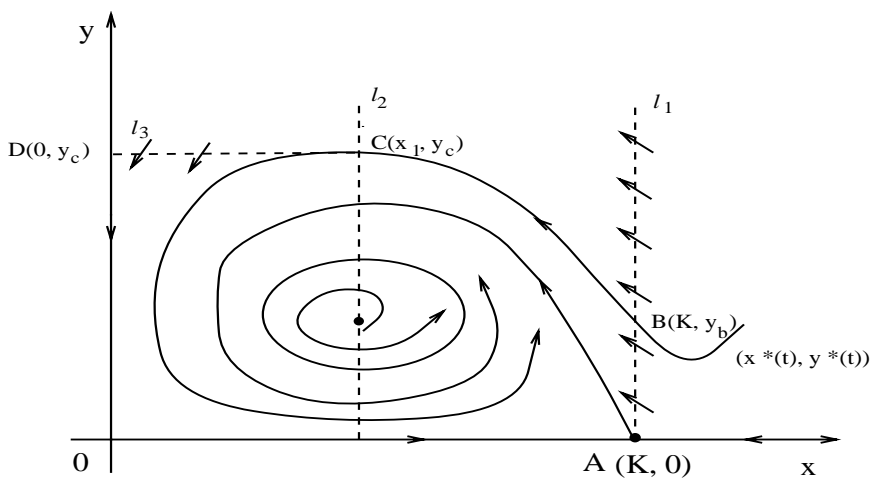

FIG. 2.5. The phase portrait of system (1.9) when $\mu^{2}>\frac{16}{3} a D^{2}$ and $x_{3}<K<x_{2}$.

Now we consider the solution $\left(x^{*}(t), y^{*}(t)\right)$ of system (1.9) passing through the point $B\left(K, y_{b}\right)$, where $y_{b}>y_{1}$. It is easy to prove that the orbit $\left(x^{*}(t), y^{*}(t)\right)$ must cross the line $l_{2}$ at point $C\left(x_{1}, y_{c}\right)$ and $y_{c} \geq y_{b}$, where $l_{2}=\left\{(x, y): x=x_{1}, y>0\right\}$.

Making a line $l_{3}=\left\{(x, y): y=y_{c}\right\}$, which begins at point $C\left(x_{1}, y_{c}\right)$ and ends at point $D\left(0, y_{c}\right)$, we can see that the direction vector field of system (1.9) on $l_{3}$ is from up to down (see Figure 2.5). Therefore the orbits of system (1.9) in the interior of the region $O A B C D O$ cannot cross the boundary.

On the other hand we know that the equilibrium $\left(x_{1}, y_{1}\right)$ is an unstable focus (or node) since $K>x_{3}$ Therefore, the existence of a periodic orbit follows directly from the Poincaré-Bendixson theorem. Moreover, the periodic orbit is stable in the interior.

THEOREM 2.5. If $\mu^{2}>\frac{16}{3} a D^{2}$ and $x_{2}>K>x_{3}$, then system (1.9) has at most one limit cycle in the interior of the first quadrant.

Proof. We prove the theorem by contradiction. First of all, we consider only the system in the domain $E_{1}$ by Theorem 2.4.

Let $x-x_{1}=-X, y-y_{1}=y_{1}\left(e^{Y}-1\right)$ and $x d t=\left(a+x^{2}\right) d T$. For the sake of simplicity, we still denote $X, Y$, and $T$ by $x, y$, and $t$, respectively. Then system (1.9) can be written as (see Zhou [40])

$$
\begin{aligned}
& \frac{d x}{d t}=\phi(y)-F(x), \\
& \frac{d y}{d t}=-g(x),
\end{aligned}
$$

where $\phi(y)=y_{1}\left(e^{y}-1\right), F(x)=r\left(1-\frac{x_{1}-x}{K}\right)\left(a+\left(x_{1}-x\right)^{2}\right)-y_{1}$, and $g(x)=$ $\frac{D x\left(x-x_{1}+x_{2}\right)}{x_{1}-x}$, where $x_{1}-K<x<x_{1}$, and $-\infty<y<+\infty$.

Note that the problem of uniqueness of a limit cycle of system (1.9) in the domain $E_{1}$ is equivalent to that of system $(2.7)$ in the domain $E_{2}$, where $E_{2}=\{(x, y)$ : $\left.x_{1}-K<x<x_{1},-\infty<y<+\infty\right\}$.

Notice that in the domain $E_{2}, \phi(y)=F(x)$ defines a smooth curve, and

$$
F(x)=\frac{r x}{K}\left(x^{2}+\left(K-3 x_{1}\right) x+a+x_{1}^{2}-2 K x_{1}+2 x_{1}^{2}\right) .
$$

When $x_{3}<K<x_{2}$, it is clear that $F(x)=0$ has three roots $r_{1}, 0$, and $r_{2}$ in $E_{2}$, where $r_{1}<0<r_{2}$. Thus, the prey-isocline $\phi(y)=F(x)$ of system (2.7) has two humps in 


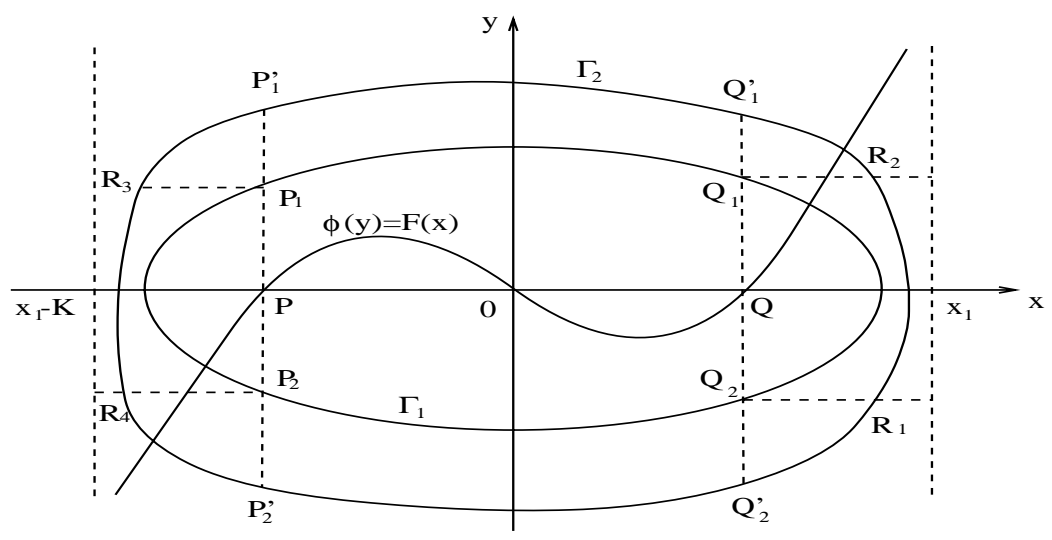

FIG. 2.6. System (2.7) has at most one limit cycle.

$E_{2}$, namely, a local maximum and a local minimum, and crosses the $x$-axis at three points, $P\left(r_{1}, 0\right), O(0,0)$, and $Q\left(r_{2}, 0\right)$, respectively.

Suppose, on the contrary, that system (2.7) has two periodic orbits $\Gamma_{1}$ and $\Gamma_{2}$; then $\Gamma_{1} \subset \operatorname{int} \Gamma_{2}$ and it is easy to prove that $\overline{P O Q} \subset \operatorname{int} \Gamma_{1}$ (see Figure 2.6).

Consider the function

$$
W(x, y)=\int_{0}^{x} g(s) d s+\int_{0}^{y} \phi(s) d s .
$$

We can see that

$$
\oint_{\Gamma_{1}} d W(x, y)=\oint_{\Gamma_{2}} d W(x, y)=0 .
$$

Now we partition $\Gamma_{1}$ and $\Gamma_{2}$ as follows (see Figure 2.6):

$$
\begin{gathered}
\Gamma_{1}=\widehat{Q_{2} Q_{1}} \bigcup \widehat{Q_{1} P_{1}} \bigcup \widehat{P_{1} P_{2}} \bigcup \widehat{P_{2} Q_{2}}, \\
\Gamma_{2}=\widehat{Q_{2}^{\prime} R_{1}} \bigcup \widehat{R_{1} R_{2}} \bigcup \widehat{R_{2} Q_{1}^{\prime}} \bigcup \widehat{Q_{1}^{\prime} P_{1}^{\prime}} \bigcup \widehat{P_{1}^{\prime} R_{3}} \bigcup \widehat{R_{3} R_{4}} \bigcup \widehat{R_{4} P_{2}^{\prime}} \bigcup \widehat{P_{2}^{\prime} Q_{2}^{\prime}} .
\end{gathered}
$$

Hence

$$
\begin{aligned}
\oint_{\Gamma_{1}} d W= & \int_{\widehat{Q_{2} Q_{1}}} d W+\int_{\widehat{Q_{1} P_{1}}} d W+\int_{\widehat{P_{1} P_{2}}} d W+\int_{\widehat{P_{2} Q_{2}}} d W, \\
\oint_{\Gamma_{2}} d W= & \int_{\widehat{Q_{2}^{\prime} R_{1}}} d W+\int_{\widehat{R_{1} R_{2}}} d W+\int_{\widehat{R_{2} Q_{1}^{\prime}}} d W+\int_{\widehat{Q_{1}^{\prime} P_{1}^{\prime}}} d W \\
& +\int_{\widehat{P_{1}^{\prime} R_{3}}} d W+\int_{\widehat{R_{3} R_{4}}} d W+\int_{\widehat{R_{4} P_{2}^{\prime}}} d W+\int_{\widehat{P_{2}^{\prime} Q_{2}^{\prime}}} d W .
\end{aligned}
$$

Since $F(x)>0$ for either $(x, y) \in \widehat{Q_{2} Q_{1}}$ or $(x, y) \in \widehat{Q_{2}^{\prime} Q_{1}^{\prime}}$, it follows that

$$
\int_{\widehat{R_{1} R_{2}}} d W=\int_{\widehat{R_{1} R_{2}}} F(x) d y>\int_{\widehat{Q_{2} Q_{1}}} d W=\int_{\widehat{Q_{2} Q_{1}}} F(x) d y,
$$




$$
\int_{\widehat{Q_{2}^{\prime} R_{1}}} d W=\int_{\widehat{Q_{2}^{\prime} R_{1}}} F(x) d y>0
$$

and

$$
\int_{\widehat{R_{2} Q_{1}^{\prime}}} d W=\int_{\widehat{R_{2} Q_{1}^{\prime}}} F(x) d y>0
$$

by the expression of $W(x, y)$ and system $(2.7)$.

On the other hand, $F(x)<0$ for either $(x, y) \in \widehat{P_{1} P_{2}}$ or $(x, y) \in \widehat{P_{1}^{\prime} P_{2}^{\prime}}$, and thus

$$
\begin{gathered}
\int_{\widehat{R_{3} R_{4}}} d W=\int_{\widehat{R_{3} R_{4}}} F(x) d y>\int_{\widehat{P_{1} P_{2}}} d W=\int_{\widehat{P_{1} P_{2}}} F(x) d y, \\
\int_{\widehat{P_{1}^{\prime} R_{3}}} d W=\int_{\widehat{P_{1}^{\prime} R_{3}}} F(x) d y>0
\end{gathered}
$$

and

$$
\int_{\widehat{R_{4} P_{2}^{\prime}}} d W=\int_{\widehat{R_{4} P_{2}^{\prime}}} F(x) d y>0
$$

However, since $F(x) g(x)<0, \phi(y)-F(x)>0$ for $(x, y) \in \widehat{Q_{1} P_{1}} \cup \widehat{Q_{1}^{\prime} P_{1}^{\prime}}$ and $F(x) g(x)<0, \phi(y)-F(x)<0$ for $(x, y) \in \widehat{P_{2} Q_{2}} \cup \widehat{P_{2}^{\prime} Q_{2}^{\prime}}$, and $\phi(y)$ is a strictly increasing function, it leads to

$$
\begin{aligned}
& \int_{\widehat{Q_{1}^{\prime} P_{1}^{\prime}}} d W=\int_{\widehat{Q_{1}^{\prime} P_{1}^{\prime}}} \frac{-F(x) g(x)}{\phi(y)-F(x)} d x>\int_{\widehat{Q_{1} P_{1}}} \frac{-F(x) g(x)}{\phi(y)-F(x)} d x=\int_{\widehat{Q_{1} P_{1}}} d W, \\
& \int_{\widehat{P_{2}^{\prime} Q_{2}^{\prime}}} d W=\int_{\widehat{P_{2}^{\prime} Q_{2}^{\prime}}} \frac{-F(x) g(x)}{\phi(y)-F(x)} d x>\int_{\widehat{P_{2} Q_{2}}} \frac{-F(x) g(x)}{\phi(y)-F(x)} d x=\int_{\widehat{P_{2} Q_{2}}} d W .
\end{aligned}
$$

Summarizing the above estimations, we have

$$
\oint_{\Gamma_{1}} d W(x, y)<\oint_{\Gamma_{2}} d W(x, y)
$$

which contradicts (2.8). Hence, system (2.7) has at most one periodic orbit. This leads to the conclusion of the theorem.

Summarizing Theorems 2.4 and 2.5, we have the following theorem.

Theorem 2.6. If $\mu^{2}>\frac{16}{3} a D^{2}$ and $x_{2}>K>x_{3}$, then system (1.9) has three equilibria: two hyperbolic saddles $(0,0)$ and $(K, 0)$ and an unstable focus (or node) $\left(x_{1}, y_{1}\right)$ in the interior of the first quadrant. Moreover, system (1.9) has a unique limit cycle, which is stable. The phase portrait is given in Figure 2.5.

TheOREM 2.7. If $x_{2}<K$ and $x_{3}<K<\frac{\mu}{D}$, then system (1.9) has at most one limit cycle in the interior of the first quadrant.

Proof. It is clear that the limit cycles of system (1.9) must be in the domain $E_{3}$ if they exist, where $E_{3}=\left\{(x, y): 0<x<x_{2}, 0<y<+\infty\right\}$.

Note that the problem of uniqueness of a limit cycle of system (1.9) in the domain $E_{3}$ is equivalent to that of system (2.7) in the domain $E_{4}$, where $E_{4}=\{(x, y)$ : $\left.x_{1}-x_{2}<x<x_{1},-\infty<y<+\infty\right\}$. 


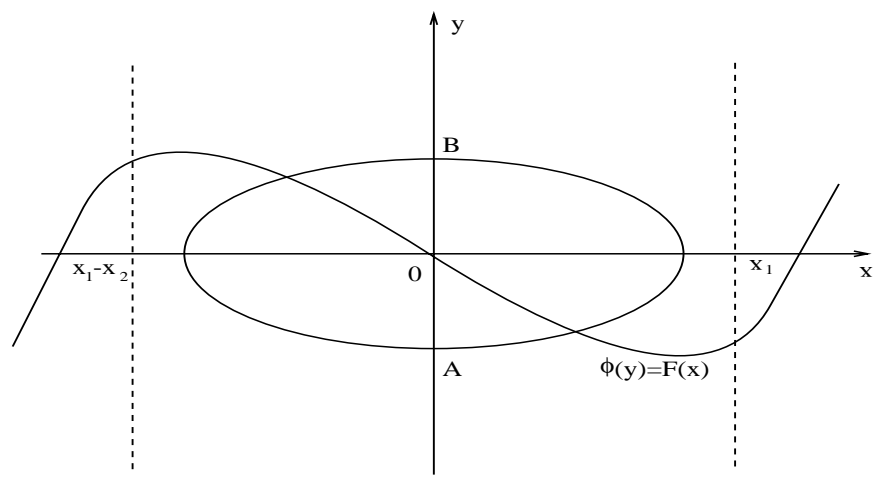

FIG. 2.7. The portrait of $\phi(y)=F(x)$ in $E_{4}$ when $K \leq \frac{\mu}{D}$.

It is clear that $\phi(y)=F(x)$ defines a smooth curve in the domain $E_{4}$, and when $x_{3}<K, F(x)=0$ has three real roots $r_{1}<0<r_{2}$ as in the proof of Theorem 2.5. Notice that since $x_{1}+x_{2}=\frac{\mu}{D}, x_{1} x_{2}=a$, and $x_{2}^{2}+a=\frac{\mu x_{2}}{D}$, we can see that as $x_{2}<K$ the three roots $r_{1}, 0, r_{2}$ of $F(x)$ are in $E_{4}$ if and only if $k<\frac{\mu}{D}$. Hence, the prey-isocline $\phi(y)=F(x)$ of system (2.7) has two humps in $E_{4}$, namely, a local maximum and a local minimum, and crosses the $x$-axis at three points, $P\left(r_{1}, 0\right), O(0,0)$, and $Q\left(r_{2}, 0\right)$, respectively. Using the same method as in the proof of Theorem 2.5, we obtain the conclusion of the theorem.

THEOREM 2.8. If $\frac{\mu}{D} \leq K$, then system (1.9) has no limit cycles in the interior of the first quadrant.

Proof. It is clear that $K>x_{2}$. Note that the problem of existence of a limit cycle of system (1.9) is equivalent to that of system (2.7) in the domain $E_{4}$.

If $K \geq \frac{\mu}{D}$, then the three roots of $F(x)=0$ satisfy $r_{1} \leq x_{1}-x_{2}<0<x_{1} \leq r_{2}$ according to the proof of Theorem 2.7. Thus, the prey-isocline $\phi(y)=F(x)$ of system (2.7) has no such humps in $E_{4}$; see Figure 2.7.

Suppose, on the contrary, that system (2.7) has a limit cycle $\Gamma$ in $E_{4}$, which crosses the $y$-axis at two points $A$ and $B$.

Consider the function

$$
W(x, y)=\int_{0}^{x} g(s) d s+\int_{0}^{y} \phi(s) d s .
$$

Then

$$
\oint_{\Gamma} d W(x, y)=0
$$

On the other hand,

$$
\oint_{\Gamma} d W(x, y)=\int_{\widehat{A B}} d W(x, y)+\int_{\widehat{B A}} d W(x, y)=\int_{\widehat{A B}} F(x) d y+\int_{\widehat{B A}} F(x) d y<0 .
$$

Obviously (2.17) contradicts (2.16). Therefore, the limit cycle $\Gamma$ does not exist. This leads to the conclusion of the theorem.

Using a similar argument as in the proof of the Theorem 2.8, we obtain the following result. 


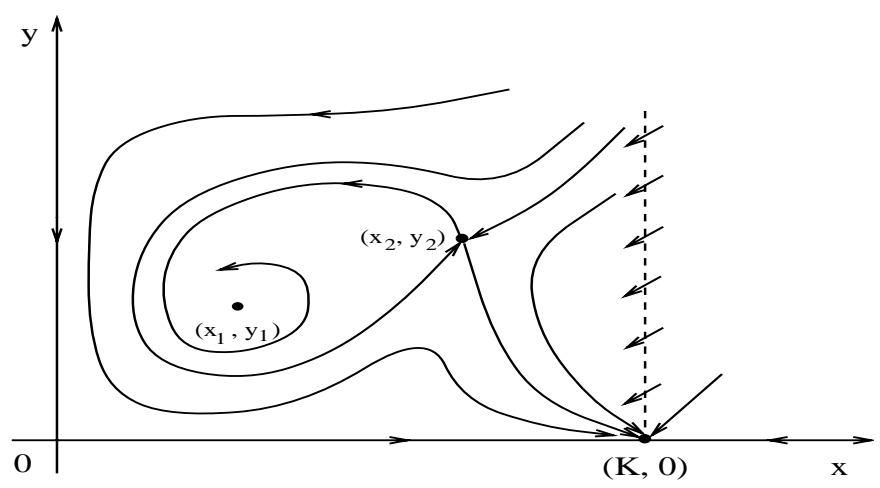

FIG. 2.8. The phase portrait of system (1.9) when $4 a D^{2}<\mu^{2}<\frac{16}{3} a D^{2}$ and $x_{2}<K<x_{3}$.

Theorem 2.9. If $\mu^{2}>\frac{16}{3} a D^{2}$ and $x_{1}<K<-x_{1}+2 \sqrt{\frac{\mu x_{1}}{D}}$, then system (1.9) has three equilibria: two hyperbolic saddles $(0,0)$ and $(K, 0)$ and a globally asymptotically stable equilibrium $\left(x_{1}, y_{1}\right)$ in the interior of the first quadrant. The phase portrait is shown in Figure 2.4.

THEOREM 2.10. If $4 a D^{2}<\mu^{2}<\frac{16}{3} a D^{2}$ and $x_{2}<K<x_{3}$, then system (1.9) has four equilibria: two hyperbolic saddles $(0,0)$ and $\left(x_{2}, y_{2}\right)$, a hyperbolic stable node $(K, 0)$, and a stable equilibrium $\left(x_{1}, y_{1}\right)$, and system (1.9) has no closed orbits. The phase portrait is given in Figure 2.8 .

Proof. We prove that system (1.9) has no closed orbits. Note that the dynamics of system (1.9) are equivalent to that of system (2.6). Therefore, we consider only system (2.6) in the domain $E_{3}=\left\{(x, y): 0<x<x_{2}, \quad 0<y<+\infty\right\}$.

Taking a Dulac function $D(x, y)=x^{-1} y^{m}$ for system (2.6), after some computations we can prove that system (2.6) has no closed orbits by the Bendixson-Dulac theorem since $\frac{K+\sqrt{K^{2}-3 a}}{3} \leq \frac{\mu-\sqrt{\mu^{2}-4 a D^{2}}}{2 D}$, where $m=\frac{2 r \sqrt{K^{2}-3 a}\left(K+\sqrt{K^{2}-3 a}\right)}{3 K \sqrt{\mu^{2}-4 a D^{2}}}-1$. Therefore, the theorem holds.

THEOREM 2.11. It is impossible that a limit cycle and a homoclinic loop of system (1.9) coexist.

Proof. Assume that system (1.9) has both a limit cycle and a homoclinic loop. Then we have that $\mu^{2}>4 a D^{2}$ and $x_{2}<K<\frac{\mu}{D}$ by Theorem 2.8. However, when $4 a D^{2}<\mu^{2} \leq \frac{16}{3} a D^{2}$ and $x_{2}<K \leq x_{3}$, system (1.9) has no limit cycles by Theorem 2.10. Therefore, $\mu^{2}>\frac{16}{3} a D^{2}$ and $x_{3}<K<\frac{\mu}{D}$. Note that the focus (or node) $\left(x_{1}, y_{1}\right)$ is unstable as $\mu^{2}>\frac{16}{3} a D^{2}$ and $x_{3}<K<\frac{\mu}{D}$. Theorem 2.7 thus implies that system (1.9) has at most one limit cycle, and it is stable if it exists.

On the other hand, if there exists a homoclinic loop, then it is stable by calculating the quantity of the saddle $\left(x_{2}, y_{2}\right)$ (i.e., the sum of the eigenvalues) as $\mu^{2}>\frac{16}{3} a D^{2}$ and $x_{3}<K<\frac{\mu}{D}$. This leads to a contradiction. Therefore, a limit cycles and a homoclinic loop cannot coexist for system (1.9).

Summarizing Theorems 2.7 and 2.11 and the supercritical Hopf bifurcation, we have the following result.

TheOrem 2.12. If $\frac{16}{3} a D^{2}<\mu^{2}<\frac{18+2 \sqrt{6}}{3} a D^{2}$ and $x_{3}<K<\frac{\mu}{D}$, then system (1.9) has four equilibria: two hyperbolic saddles $(0,0)$ and $\left(x_{2}, y_{2}\right)$, a hyperbolic stable node $(K, 0)$, and an unstable focus (or node) $\left(x_{1}, y_{1}\right)$. Moreover, there exists a constant $\epsilon_{0}>0$ such that the phase portrait of system (1.9) can be sketched in Figure 2.9 as $\frac{18+2 \sqrt{6}}{3} a D^{2}>\mu^{2}>\frac{16}{3} a D^{2}$ and $x_{3}<K<x_{3}+\epsilon_{0}<\frac{\mu}{D}$ (i.e., there exists a unique 


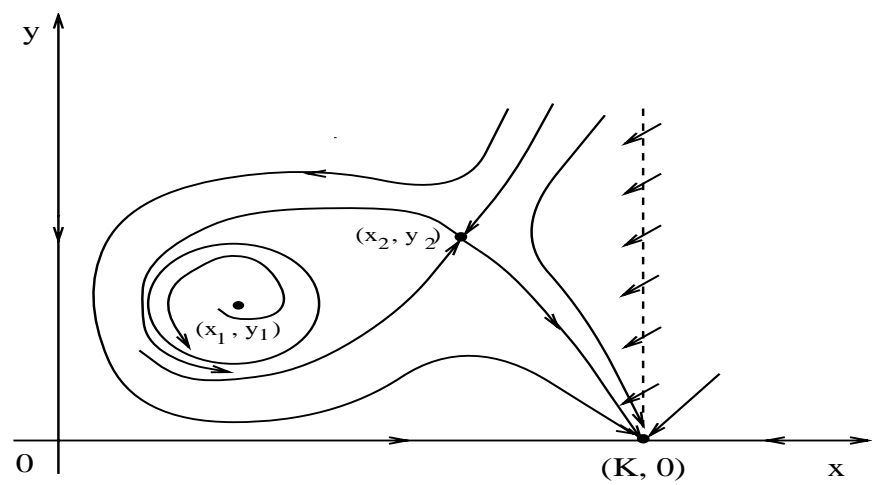

FIG. 2.9. The phase portrait of system (1.9) when $\frac{18+2 \sqrt{6}}{3} a D^{2}>\mu>\frac{16}{3} a D^{2}$ and $x_{3}<K<$ $x_{3}+\epsilon_{0}$.

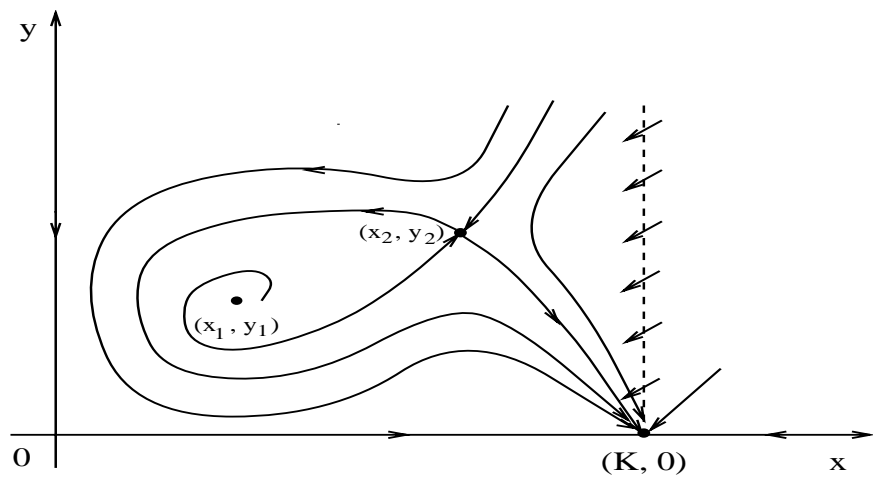

FIG. 2.10. The phase portrait of system (1.9) when $\mu^{2}>4 a D^{2}$ and $K>x_{4}$.

limit cycle, which is stable).

According to Theorem 2.8, we obtain the following theorem by calculating the quantity of the saddle $\left(x_{2}, y_{2}\right)$.

THEOREM 2.13. If $\mu^{2}>4 a D^{2}$ and $K>x_{4}$, then system (1.9) has four equilibria: two hyperbolic saddles $(0,0)$ and $\left(x_{2}, y_{2}\right)$, a hyperbolic stable node $(K, 0)$, and an unstable equilibrium $\left(x_{1}, y_{1}\right)$. Moreover, system (1.9) has no closed orbits, that is, it exhibits the so-called paradox of enrichment phenomenon. The phase portrait is given in Figure 2.10.

Remark 2.14. When $\frac{18+2 \sqrt{6}}{3} a D^{2} \leq \mu^{2}$ and $K \leq x_{3}$, the dynamics of system (1.9) in the interior of the first quadrant could be very complicated. There may exist two or more limit cycles.

Remark 2.15. In section 3, we will show that system (1.9) has a homoclinic loop when parameters take some suitable values.

Remark 2.16. We summarize and classify the global dynamics of system (1.9) in the interior of the first quadrant by dividing $4 a D^{2}<\mu^{2}$ into two cases:

(I) $4 a D^{2}<\mu^{2} \leq \frac{16}{3} a D^{2}$ (see Table 2.1);

(II) $\frac{16}{3} a D^{2}<\mu^{2}$ (see Table 2.2).

Case (II) can be further classified into three subcases:

(II $) \frac{16}{3} a D^{2}<\mu^{2}<\frac{18+2 \sqrt{6}}{3} a D^{2}$; 
TABLE 2.1

$4 a D^{2}<\mu^{2} \leq \frac{16}{3} a D^{2}$.

\begin{tabular}{|c|l|}
\hline Range of parameter $K$ & Number of interior equilibria and dynamics of (1.9) \\
\hline$x_{1}<K \leq x_{2}$ & one equilibrium: $\left(x_{1}, y_{1}\right)$ globally stable focus (or node) \\
\hline$x_{2}<K<x_{3}$ & $\begin{array}{l}\text { two equilibria: }\left(x_{1}, y_{1}\right) \text { stable focus (or node) }\left(x_{2}, y_{2}\right) \text { saddle } \\
\text { no closed orbit (neither limit cycle nor homoclinic loop) }\end{array}$ \\
\hline$K=x_{3}$ & $\begin{array}{l}\text { two equilibria: }\left(x_{1}, y_{1}\right) \text { stable multiple focus with multiplicity 1; } \\
\left(x_{2}, y_{2}\right) \text { saddle; no closed orbit }\end{array}$ \\
\hline & $\begin{array}{l}\left.\text { two equilibria: ( } x_{1}, y_{1}\right) \text { unstable focus; }\left(x_{2}, y_{2}\right) \text { saddle } \\
\text { supercritical Hopf bifurcation } \\
\text { if a limit cycle exists, it is unique and stable } \\
\text { if a homoclinic loop exists, it is stable } \\
\text { a limit cycle and a homoclinic loop cannot coexist }\end{array}$ \\
\hline$x_{3}<K<\frac{\mu}{D}$ & $\begin{array}{l}\text { two equilibria: }\left(x_{1}, y_{1}\right) \text { unstable focus; }\left(x_{2}, y_{2}\right) \text { saddle } \\
\text { no limit cycle }\end{array}$ \\
\hline$\frac{\mu}{D} \leq K \leq x_{4}$ & $\begin{array}{l}\text { two equilibria: }\left(x_{1}, y_{1}\right) \text { unstable focus; }\left(x_{2}, y_{2}\right) \text { saddle } \\
\text { no closed orbit }\end{array}$ \\
\hline
\end{tabular}

TABLE 2.2

$\frac{16}{3} a D^{2}<\mu^{2}$.

\begin{tabular}{|c|c|}
\hline Range of parameter $K$ & Number of interior equilibria and dynamics of (1.9) \\
\hline$x_{1}<K<-x_{1}+2 \sqrt{\frac{\mu x_{1}}{D}}$ & one equilibrium: $\left(x_{1}, y_{1}\right)$ globally stable focus (or node) \\
\hline$-x_{1}+2 \sqrt{\frac{\mu x_{1}}{D}} \leq K<x_{3}$ & $\begin{array}{l}\text { one equilibrium: }\left(x_{1}, y_{1}\right) \text { stable focus (or node) } \\
\left(\mathrm{II}_{3}\right) \text { : subcritical Hopf bifurcation; if there exist limit cycles, } \\
\text { there is at least one which is unstable from inside }\end{array}$ \\
\hline$K=x_{3}$ & $\begin{array}{l}\text { one equilibrium }\left(x_{1}, y_{1}\right) \\
\left(\mathrm{II}_{1}\right): \text { stable multiple focus with multiplicity } 1 \\
\left(\mathrm{II}_{2}\right): \text { multiple focus with multiplicity at least } 2 \\
\left(\mathrm{II}_{3}\right): \text { unstable multiple focus with multiplicity } 1\end{array}$ \\
\hline$x_{3}<K \leq x_{2}$ & $\begin{array}{l}\left(\mathrm{II}_{1}\right) \text { : one equilibrium }\left(x_{1}, y_{1}\right) \text {, unstable focus (or node) } \\
\text { supercritical Hopf bifurcation } \\
\text { a unique limit cycle exists and is stable } \\
\left(\mathrm{II}_{2,3}\right) \text { : one equilibrium: }\left(x_{1}, y_{1}\right) \text { unstable focus (or node) } \\
\text { there exists a stable unique limit cycle }\end{array}$ \\
\hline$x_{2}<K<\frac{\mu}{D}$ & $\begin{array}{l}\text { two equilibria: }\left(x_{1}, y_{1}\right) \text { unstable focus; }\left(x_{2}, y_{2}\right) \text { saddle } \\
\text { if a limit cycle exists, it is unique and stable } \\
\text { if a homoclinic loop exists, it is stable } \\
\text { a limit cycle and a homoclinic loop cannot coexist }\end{array}$ \\
\hline$\frac{\mu}{D} \leq K \leq x_{4}$ & $\begin{array}{l}\text { two equilibria: }\left(x_{1}, y_{1}\right) \text { unstable focus; }\left(x_{2}, y_{2}\right) \text { saddle } \\
\text { no limit cycle }\end{array}$ \\
\hline$x_{4}<K$ & $\begin{array}{l}\text { two equilibria: }\left(x_{1}, y_{1}\right) \text { unstable focus; }\left(x_{2}, y_{2}\right) \text { saddle } \\
\text { no closed orbit }\end{array}$ \\
\hline
\end{tabular}

(II $)_{2} \mu^{2}=\frac{18+2 \sqrt{6}}{3} a D^{2}$;

(II $) \frac{18+2 \sqrt{6}}{3} a D^{2}<\mu^{2}$.

In each case, the number of interior equilibria and the dynamics vary as $K$, the carrying capacity, takes different values.

3. Bifurcations of the degenerate singular point. From the analysis in section 2, we know that when $\mu=K D$ and $\mu^{2}-4 a D^{2}=0$, system (1.9) has an interior equilibrium $\left(x_{0}, y_{0}\right)$ which is a cusp. In this section, we are interested in the bifurcation of the cusp as the parameters vary in a small neighborhood of $\left(\mu_{0}, K_{0}, a_{0}, D_{0}\right)$, where 
$\mu_{0}, K_{0}, a_{0}$, and $D_{0}$ satisfy the equations

$$
\begin{aligned}
& \mu-K D=0, \\
& \mu^{2}-4 a D^{2}=0 .
\end{aligned}
$$

By bifurcation analysis we show that system (1.9) has a homoclinic loop for some parameters values. Now we consider the system

$$
\begin{aligned}
& \dot{x}=r x\left(1-\frac{x}{K_{0}}\right)-\frac{x y}{a_{0}+x^{2}}, \\
& \dot{y}=y\left(\frac{\mu_{0} x}{a_{0}+x^{2}}-D_{0}\right) .
\end{aligned}
$$

First of all, we translate the interior equilibrium $\left(x_{0}, y_{0}\right)$ to the origin and expand system (3.3) in a power series around the origin. Let $X=x-x_{0}, Y=y-y_{0}$. Then system (3.3) can be rewritten as

$$
\begin{aligned}
\dot{X} & =-\frac{x_{0}}{a_{0}+x_{0}^{2}} Y+\left(\frac{x_{0} y_{0}}{\left(a_{0}+x_{0}^{2}\right)^{2}}-\frac{r}{K_{0}}\right) X^{2}+P_{1}(X, Y), \\
\dot{Y} & =-\frac{\mu_{0} x_{0} y_{0}}{\left(a_{0}+x_{0}^{2}\right)^{2}} X^{2}+Q_{1}(X, Y)
\end{aligned}
$$

where $x_{0}=\frac{\mu_{0}}{2 D_{0}}, y_{0}=r\left(1-\frac{x_{0}}{K_{0}}\right)\left(a_{0}+x_{0}^{2}\right)$, and $P_{1}$ and $Q_{1}$ are $C^{\infty}$ functions in $(X, Y)$ at least of the third order. Next, we study the normal form of the system (3.4) in the two-dimensional center manifold. Making the affine transformation

$$
x=X, y=-\frac{x_{0}}{a_{0}+x_{0}^{2}} Y,
$$

we can rewrite system (3.4) as follows:

$$
\begin{aligned}
& \dot{x}=y+\left(\frac{x_{0} y_{0}}{\left(a_{0}+x_{0}^{2}\right)^{2}}-\frac{r}{K_{0}}\right) x^{2}+\tilde{P}_{1}(x, y), \\
& \dot{y}=\frac{\mu_{0} x_{0}^{2} y_{0}}{\left(a_{0}+x_{0}^{2}\right)^{3}} x^{2}+\tilde{Q}_{1}(x, y) .
\end{aligned}
$$

In order to find the canonical normal form of the cusp, we take

$$
X=x, \quad Y=y+\left(\frac{x_{0} y_{0}}{\left(a_{0}+x_{0}^{2}\right)^{2}}-\frac{r}{K_{0}}\right) x^{2}+\tilde{P}_{1}(x, y) .
$$

Then system (3.5) becomes

$$
\begin{aligned}
& \dot{X}=Y, \\
& \dot{Y}=\frac{\mu_{0} x_{0}^{2} y_{0}}{\left(a_{0}+x_{0}^{2}\right)^{3}} X^{2}+2\left(\frac{x_{0} y_{0}}{\left(a_{0}+x_{0}^{2}\right)^{2}}-\frac{r}{K_{0}}\right) X Y+R(X, Y),
\end{aligned}
$$

where $R$ is a $C^{\infty}$ function in $(X, Y)$ at least of the third order. Since

$$
\frac{2 x_{0} y_{0}}{\left(a_{0}+x_{0}^{2}\right)^{2}}-\frac{2 r}{K_{0}}=-\frac{D_{0}}{\mu_{0}} r<0, \quad \frac{\mu_{0} x_{0}^{2} y_{0}}{\left(a_{0}+x_{0}^{2}\right)^{3}}=\frac{r \mu_{0}}{8 a_{0}}>0
$$


we have the following theorem.

THEOREM 3.1. The interior equilibrium $\left(x_{0}, y_{0}\right)$ of system (3.3) is a cusp of codimension 2 .

The above theorem indicates that system (3.3) could exhibit the BogdanovTakens bifurcation under a small parameter perturbation if the bifurcation parameters are chosen suitably. In the following, we will see what parameters can be chosen as bifurcation parameters. For convenience, we denote

$$
\left(\mu_{0}, K_{0}, a_{0}, D_{0}\right)=\left(\lambda_{1}^{0}, \lambda_{2}^{0}, \lambda_{3}^{0}, \lambda_{4}^{0}\right)=\lambda^{0}, \quad(\mu, K, a, D)=\left(\lambda_{1}, \lambda_{2}, \lambda_{3}, \lambda_{4}\right)=\lambda .
$$

Then system (1.9) in a small neighborhood of $\left(x_{0}, y_{0}\right)$ can be rewritten as

$$
\begin{aligned}
& \dot{x}=y+W_{1}(x, y, \lambda, r), \\
& \dot{y}=\frac{r \mu_{0}}{8 a_{0}} x^{2}-\frac{D_{0}}{\mu_{0}} r x y+W_{2}(x, y, \lambda, r),
\end{aligned}
$$

where $W_{1}, W_{2} \in C^{\infty}\left(R^{2} \times R^{5}, R\right), W_{1}\left(x, y, \lambda_{0}, r\right)=0, W_{2}\left(x, y, \lambda_{0}, r\right)=R(x, y), x$ and $y$ belong to a small neighborhood of $(0,0)$, and $\lambda$ is in a small neighborhood of $\lambda_{0}$.

Lemma 3.2. System (3.7) is $C^{\infty}$ equivalent to

$$
\begin{aligned}
& \dot{x}=y, \\
& \dot{y}=\phi(\lambda, r)+\psi(\lambda, r) x+x^{2}+\left(-6 D_{0} \sqrt{\frac{a_{0} r}{\mu_{0}}}+\alpha(\lambda, r)\right) x y+\tilde{R}(x, y, \lambda, r),
\end{aligned}
$$

where $\phi, \psi$, and $\alpha$ are smooth functions, $\phi\left(\lambda_{0}, r\right)=\psi\left(\lambda_{0}, r\right)=0, \alpha\left(\lambda_{0}, r\right)=0, \tilde{R}$ is a $C^{\infty}$ function in $(x, y)$ at least of the third order.

Proof. Making the parameter-dependent nonsingular change of variables

$$
X=x, Y=y+W_{1}(x, y, \lambda, r),
$$

then system (3.7) can be written as

$$
\begin{aligned}
\dot{X} & =Y, \\
\dot{Y} & =P(X, \lambda, r)+Y F(X, \lambda, r)+Y^{2} G(X, Y, \lambda, r),
\end{aligned}
$$

where $P, F, G \in C^{\infty}$ and

$$
\begin{aligned}
& P\left(0, \lambda_{0}, r\right)=\frac{\partial P\left(0, \lambda_{0}, r\right)}{\partial X}=0, \quad \frac{\partial^{2} P\left(0, \lambda_{0}, r\right)}{\partial X^{2}}=\frac{r \mu_{0}}{8 a_{0}}>0, \\
& F\left(0, \lambda_{0}, r\right)=0, \quad \frac{\partial F\left(0, \lambda_{0}, r\right)}{\partial X}=-\frac{D_{0}}{\mu_{0}} r<0, \quad G\left(0,0, \lambda_{0}, r\right)=0 .
\end{aligned}
$$

Applying the Malgrange preparation theorem (see [7]) to the function $P(X, \lambda, r)$, we have

$$
P(X, \lambda, r)=\left[\phi(\lambda, r)+\psi(\lambda, r) X+X^{2}\right] B(X, \lambda, r),
$$

where $\phi, \psi$ and $B \in C^{\infty}, B\left(0, \lambda_{0}, r\right)=\frac{r \mu_{0}}{8 a_{0}} \neq 0, \phi\left(\lambda_{0}, r\right)=\psi\left(\lambda_{0}, r\right)=0$. Thus, system (3.9) can be rewritten as

$$
\begin{aligned}
& \dot{X}=Y, \\
& \dot{Y}=\left[\phi(\lambda, r)+\psi(\lambda, r) X+X^{2}+\frac{Y F(X, \lambda, r)}{B(X, \lambda, r)}+\frac{Y^{2} G(X, Y, \lambda, r)}{B(X, \lambda, r)}\right] B(X, \lambda, r) .
\end{aligned}
$$


Let

$$
x=X, \quad y=\frac{Y}{\sqrt{B(X, \lambda, r)}}, \quad \tau=\int_{0}^{t} \sqrt{B(X(s), \lambda, r)} d s .
$$

Then system (3.10) becomes

$$
\begin{aligned}
& \dot{x}=y, \\
& \dot{y}=\phi(\lambda, r)+\psi(\lambda, r) x+x^{2}+\frac{y F(x, \lambda, r)}{\sqrt{B(x, \lambda, r)}}+y^{2} \tilde{G}(x, y, \lambda, r),
\end{aligned}
$$

where $\dot{x}=\frac{d x}{d \tau}$ and $\dot{y}=\frac{d y}{d \tau}$. Expanding the function $\frac{F(x, \lambda, r)}{\sqrt{B(x, \lambda, r)}}$ in a power series around $x=0$, we have

$$
\frac{F(x, \lambda, r)}{\sqrt{B(x, \lambda, r)}}=\left(-\sqrt{\frac{2 r}{\mu_{0}}}+\alpha(\lambda, r)\right) x+\tilde{F}(x, \lambda, r),
$$

where $\alpha\left(\lambda_{0}, r\right)=0$, and $\tilde{F}$ is a $C^{\infty}$ function in $x$ at least of the second order. Denote

$$
\tilde{R}(x, y, \lambda, r)=y \tilde{F}(x, \lambda, r)+y^{2} \tilde{G}(x, y, \lambda, r) .
$$

Then system (3.11) is transformed into system (3.8).

THEOREM 3.3. If the rank of the matrix

$$
N=\left(\begin{array}{lllll}
\frac{\partial \phi_{1}(\lambda, r)}{\partial r} & \frac{\partial \phi_{1}(\lambda, r)}{\partial \lambda_{1}} & \frac{\partial \phi_{1}(\lambda, r)}{\partial \lambda_{2}} & \frac{\partial \phi_{1}(\lambda, r)}{\partial \lambda_{3}} & \frac{\partial \phi_{1}(\lambda, r)}{\partial \lambda_{4}} \\
\frac{\partial \phi_{2}(\lambda, r)}{\partial r} & \frac{\partial \phi_{2}(\lambda, r)}{\partial \lambda_{1}} & \frac{\partial \phi_{2}(\lambda, r)}{\partial \lambda_{2}} & \frac{\partial \phi_{2}(\lambda, r)}{\partial \lambda_{3}} & \frac{\partial \phi_{2}(\lambda, r)}{\partial \lambda_{4}}
\end{array}\right)_{\left(\lambda_{0}, r\right)}
$$

is 2, then we can choose bifurcation parameters such that system (3.7) exhibits the Bogdanov-Takens bifurcation.

Proof. Since the rank of the matrix $N$ is 2, we can choose two parameters, either $\left(\lambda_{i}, \lambda_{j}\right)$ or $\left(r, \lambda_{i}\right)$, in $(\lambda, r)$ such that the determinant of either the matrix

$$
N_{1}=\left(\begin{array}{ll}
\frac{\partial \phi_{1}(\lambda, r)}{\partial \lambda_{i}} & \frac{\partial \phi_{1}(\lambda, r)}{\partial \lambda_{j}} \\
\frac{\partial \phi_{2}(\lambda, r)}{\partial \lambda_{i}} & \frac{\partial \phi_{2}(\lambda, r)}{\partial \lambda_{j}}
\end{array}\right)_{\left(\lambda_{0}, r\right)}
$$

or the matrix

$$
N_{2}=\left(\begin{array}{ll}
\frac{\partial \phi_{1}(\lambda, r)}{\partial r} & \frac{\partial \phi_{1}(\lambda, r)}{\partial \lambda_{j}} \\
\frac{\partial \phi_{2}(\lambda, r)}{\partial r} & \frac{\partial \phi_{2}(\lambda, r)}{\partial \lambda_{j}}
\end{array}\right)_{\left(\lambda_{0}, r\right)}
$$

is not zero. Without loss of generality, assume that the determinant of $N_{1}$ is not zero. Then we choose the parameters $\lambda_{i}$ and $\lambda_{j}$ as bifurcation parameters. Let

$$
\mu=\phi_{1}(\lambda, r), \nu=\phi_{2}(\lambda, r), \eta_{1}=r, \eta_{2}=\lambda_{k}, \eta_{3}=\lambda_{l}, l, k \neq i, j .
$$

Obviously, the above parameters transformation is not singular. Hence, system (3.8) can be written as

$$
\begin{aligned}
& \dot{x}=y, \\
& \dot{y}=\mu+\nu x+x^{2}-\sqrt{\frac{2 r}{\mu_{0}}} x y+\tilde{\tilde{R}}\left(x, y, \mu, \nu, \eta_{1}, \eta_{2}, \eta_{3}\right),
\end{aligned}
$$


where $\tilde{\tilde{R}}$ is a $C^{\infty}$ function in $(x, y)$ at least of the second order and the coefficient of the second order is zero if $\left(\mu, \nu, \eta_{1}, \eta_{2}, \eta_{3}\right)=\left(\phi_{1}\left(\lambda_{0}, r\right), \phi_{2}\left(\lambda_{0}, r\right), r, \lambda_{k}^{0}, \lambda_{l}^{0}\right)$. By the theorems in [4], [5], and [33], we know that the universal unfoldings (or versal deformation) of system (3.13) is

$$
\begin{aligned}
& \dot{x}=y, \\
& \dot{y}=\mu+\nu x+x^{2}-\sqrt{\frac{2 r}{\mu_{0}}} x y .
\end{aligned}
$$

Therefore, system (3.13) exhibits the Bogdanov-Takens bifurcation.

Remark 3.4. When the rank of the matrix $N$ equals 2, we say that system (3.7) has the generic property, i.e., system (3.7) is a generic family.

Remark 3.5. From the above analysis, we can see that the parameter $r$ cannot be taken as a bifurcation parameter. In fact, $\operatorname{det}\left(N_{2}\right)=0$.

THEOREM 3.6. If system (3.7) is a generic family, then there exists a unique smooth surface $H L$ corresponding to the homoclinic bifurcation and a unique smooth surface $H$ corresponding to the Hopf bifurcation such that system (3.7) has a unique and hyperbolic stable cycle for parameter values inside the region bounded by $H$ and $H L$ and no cycles outside this region.

The theorem can be proved directly from the results in [4], [5], and [33] by the linear transformation of coordinates $(x, y, t, \mu, \nu) \rightarrow(x,-y,-t, \mu, \nu)$. In the above analysis, we chose two parameters as bifurcation parameters so that the predator-prey system with group defense exhibits the Bogdanov-Takens bifurcation. By Theorem 3.1 , the system cannot have bifurcations with codimension greater than 2. Therefore, the existence of a semistable periodic orbit as well as the coexistence of a limit cycle and a homoclinic loop cannot occur for the generic system (3.7). However, if the functional response function $p(x)=\frac{x}{a+b x+x^{2}}$, where $a$ and $b$ are positive constants, then these phenomena can occur if we choose three suitable bifurcation parameters, that is, there are bifurcations of codimension 3. See also Rothe and Shafer [30].

Finally, we choose $K$ and $D$ as bifurcation parameters and make a bifurcation analysis of system (1.9) depending on these two parameters. We will show that system (1.9) has a homoclinic loop and sketch the bifurcation diagram and the corresponding phase portraits. Consider

$$
\begin{aligned}
& \dot{x}=r x\left(1-\frac{x}{K_{0}+\lambda_{1}}\right)-\frac{x y}{a_{0}+x^{2}}, \\
& \dot{y}=y\left(\frac{\mu_{0} x}{a_{0}+x^{2}}-D_{0}-\lambda_{2}\right),
\end{aligned}
$$

where the constants $\mu_{0}, K_{0}, a_{0}$, and $D_{0}$ are described in section $3, r$ is a positive constant, and $\lambda_{1}$ and $\lambda_{2}$ are parameters in a small neighborhood of $(0,0)$. We are interested only in the phase portraits of system (3.15) when $x$ and $y$ are in a small neighborhood of the interior equilibrium $\left(x_{0}, y_{0}\right)=\left(\frac{\mu_{0}}{2 D_{0}}, r a_{0}\right)$. We translate $\left(x_{0}, y_{0}\right)$ to the origin and expand system (3.15) in a power series around the origin. Let

$$
\bar{x}=x-x_{0}, \quad \bar{y}=y-y_{0} .
$$


Then we have

$$
\begin{aligned}
\dot{\bar{x}}= & \frac{r}{4} \lambda_{1}+b_{1}\left(\lambda_{1}\right)+\left[\frac{x_{0} r}{2 a_{0}} \lambda_{1}+b_{2}\left(\lambda_{1}\right)\right] \bar{x}-\frac{x_{0}}{2 a_{0}} \bar{y} \\
& +\left[\frac{x_{0} y_{0}}{4 a_{0}^{2}}-\frac{r}{K_{0}}+\frac{r}{4 a_{0}} \lambda_{1}+b_{3}\left(\lambda_{1}\right)\right] \bar{x}^{2}+B\left(\bar{x}, \bar{y}, \lambda_{1}\right), \\
\dot{\bar{y}}= & -y_{0} \lambda_{2}-\lambda_{2} \bar{y}+\frac{\mu_{0} x_{0}^{3} y_{0}-3 \mu_{0} a_{0} x_{0} y_{0}}{\left(a_{0}+x_{0}^{2}\right)^{3}} \bar{x}^{2}+C\left(\bar{x}, \bar{y}, \lambda_{2}\right),
\end{aligned}
$$

where $b_{i}\left(\lambda_{1}\right)(i=1,2,3)$ are smooth functions at least of the second order, $B$ and $C$ are $C^{\infty}$ functions at least of the third order with respect to $(\bar{x}, \bar{y})$, and the coefficients depend smoothly on $\lambda_{1}$ and $\lambda_{2}$, respectively. Hereafter, we assume that these transformations have been made and drop the overbars. System (3.16) can be written as

$$
\begin{aligned}
\dot{x}= & \frac{r}{4} \lambda_{1}+b_{1}\left(\lambda_{1}\right)+\left(\frac{x_{0} r}{2 a_{0}} \lambda_{1}+b_{2}\left(\lambda_{1}\right)\right) x-\frac{x_{0}}{2 a_{0}} y \\
& +\left[\frac{x_{0} y_{0}}{4 a_{0}^{2}}-\frac{r}{K_{0}}+\frac{r}{4 a_{0}} \lambda_{1}+b_{3}\left(\lambda_{1}\right)\right] x^{2}+B\left(x, y, \lambda_{1}\right), \\
\dot{y}= & -y_{0} \lambda_{2}-\lambda_{2} y-\frac{\mu_{0} x_{0} y_{0}}{\left(a_{0}+x_{0}^{2}\right)^{2}} x^{2}+C\left(x, y, \lambda_{2}\right) .
\end{aligned}
$$

Making the parameter-dependent affine transformation

$$
X=x, \quad Y=\left[\frac{x_{0} r}{2 a_{0}} \lambda_{1}+b_{2}\left(\lambda_{1}\right)\right] x-\frac{x_{0}}{2 a_{0}} y,
$$

we can see that system (3.17) becomes

$$
\begin{aligned}
\dot{X}= & \frac{r}{4} \lambda_{1}+b_{1}\left(\lambda_{1}\right)+Y+2\left[\frac{x_{0} y_{0}}{4 a_{0}^{2}}-\frac{r}{K_{0}}+\frac{r}{4 a_{0}} \lambda_{1}+b_{3}\left(\lambda_{1}\right)\right] X^{2}+\tilde{B}\left(X, Y, \lambda_{1}\right), \\
\dot{Y}= & \frac{x_{0} y_{0}}{2 a_{0}} \lambda_{2}+\left(\frac{r}{4} \lambda_{1}+b_{1}\left(\lambda_{1}\right)\right)\left(\frac{x_{0} r}{2 a_{0}} \lambda_{1}+b_{2}\left(\lambda_{1}\right)\right) \\
& +\left(\frac{x_{0} r}{2 a_{0}} \lambda_{1}-\lambda_{2}+b_{2}\left(\lambda_{1}\right)\right) Y+\lambda_{2}\left(\frac{x_{0} r}{2 a_{0}} \lambda_{1}+b_{2}\left(\lambda_{1}\right)\right) X \\
& +\left[\frac{\mu_{0} x_{0}^{2} y_{0}}{2 a_{0}\left(a_{0}+x_{0}^{2}\right)^{2}}+2\left(\frac{x_{0} r}{2 a_{0}} \lambda_{1}+b_{2}\left(\lambda_{1}\right)\right)\left(\frac{x_{0} y_{0}}{4 a_{0}^{2}}-\frac{r}{K_{0}}+\frac{r}{4 a_{0}} \lambda_{1}+b_{3}\left(\lambda_{1}\right)\right)\right] X^{2} \\
& +\tilde{C}\left(X, Y, \lambda_{1}, \lambda_{2}\right) \\
= & \frac{x_{0} y_{0}}{2 a_{0}} \lambda_{2}+c_{1}\left(\lambda_{1}\right)+\left(\frac{x_{0} r}{2 a_{0}} \lambda_{1}-\lambda_{2}+b_{2}\left(\lambda_{1}\right)\right) Y+c_{2}\left(\lambda_{1}, \lambda_{2}\right) X \\
& +\left[\frac{\mu_{0} x_{0}^{2} y_{0}}{2 a_{0}\left(a_{0}+x_{0}^{2}\right)^{2}}+c_{3}\left(\lambda_{1}\right)\right] X^{2}+\tilde{C}\left(X, Y, \lambda_{1}, \lambda_{2}\right),
\end{aligned}
$$

where $\tilde{B}$ and $\tilde{C}$ are $C^{\infty}$ functions in variables $(X, Y)$ at least of the third order, the coefficients depend smoothly on $\lambda_{1}$ and $\lambda_{2}$, and $c_{1}, c_{2}$, and $c_{3}$ are smooth functions of their variables. Let

$$
\begin{aligned}
& x=X, \\
& y=\frac{r}{4} \lambda_{1}+b_{1}\left(\lambda_{1}\right)+Y+2\left[\frac{x_{0} y_{0}}{4 a_{0}^{2}}-\frac{r}{K_{0}}+\frac{r}{4 a_{0}} \lambda_{1}+b_{3}\left(\lambda_{1}\right)\right] X^{2}+\tilde{B}\left(X, Y, \lambda_{1}\right) .
\end{aligned}
$$


Then system (3.18) can be written as

$$
\begin{aligned}
\dot{x}= & y \\
\dot{y}= & \frac{x_{0} y_{0}}{2 a_{0}} \lambda_{2}+\tilde{c}_{1}\left(\lambda_{1}, \lambda_{2}\right)+\left(\frac{x_{0} r}{2 a_{0}} \lambda_{1}-\lambda_{2}+b_{2}\left(\lambda_{1}\right)\right) y+\tilde{c}_{2}\left(\lambda_{1}, \lambda_{2}\right) x \\
& +\left[\frac{\mu_{0} x_{0}^{2} y_{0}}{2 a_{0}\left(a_{0}+x_{0}^{2}\right)^{2}}+\tilde{c}_{3}\left(\lambda_{1}, \lambda_{2}\right)\right] x^{2}+2\left[\frac{x_{0} y_{0}}{4 a_{0}^{2}}-\frac{r}{K_{0}}+\frac{r}{4 a_{0}} \lambda_{1}+b_{3}\left(\lambda_{1}\right)\right] x y \\
& +\tilde{R}\left(x, y, \lambda_{1}, \lambda_{2}\right) \\
= & \frac{x_{0} y_{0}}{2 a_{0}} \lambda_{2}+\tilde{c}_{1}\left(\lambda_{1}, \lambda_{2}\right)+\left(\frac{x_{0} r}{2 a_{0}} \lambda_{1}-\lambda_{2}+b_{2}\left(\lambda_{1}\right)\right) y+\tilde{c}_{2}\left(\lambda_{1}, \lambda_{2}\right) x \\
& +\left[\frac{\mu_{0} r}{8 a_{0}}+\tilde{c}_{3}\left(\lambda_{1}, \lambda_{2}\right)\right] x^{2}-\left[\frac{r}{2 x_{0}}-\frac{r}{4 a_{0}} \lambda_{1}-b_{3}\left(\lambda_{1}\right)\right] x y+\tilde{R}\left(x, y, \lambda_{1}, \lambda_{2}\right),
\end{aligned}
$$

where $\tilde{c}_{i}(i=1,2,3)$ are smooth functions of $\left(\lambda_{1}, \lambda_{2}\right), \tilde{R}$ is a $C^{\infty}$ function in variables $\left(\lambda_{1}, \lambda_{2}\right)$ and at least of the third order with respect to $(x, y)$, and the coefficients depend smoothly on $\lambda_{1}$ and $\lambda_{2}$. Rewriting system (3.19) as the form in system (3.9) and using the method in the proof of Lemma 3.1, we obtain

$$
\begin{aligned}
\dot{x}= & y, \\
\dot{y}= & \frac{4 a_{0} x_{0}}{\mu_{0}} \lambda_{2}+\phi_{1}\left(\lambda_{1}, \lambda_{2}\right)+\phi_{2}\left(\lambda_{1}, \lambda_{2}\right) x+\left[\frac{\frac{x_{0} r}{2 a_{0}} \lambda_{1}-\lambda_{2}}{\sqrt{\frac{\mu_{0} r}{8 a_{0}}}}+\phi_{3}\left(\lambda_{1}, \lambda_{2}\right)\right] y \\
& +x^{2}-\left[\frac{\frac{r}{2 x_{0}}}{\sqrt{\frac{\mu_{0} r}{8 a_{0}}}}+\phi_{4}\left(\lambda_{1}, \lambda_{2}\right)\right] x y+R\left(x, y, \lambda_{1}, \lambda_{2}\right),
\end{aligned}
$$

where $\phi_{1}, \phi_{2}$, and $\phi_{3}$ are smooth functions in variables $\left(\lambda_{1}, \lambda_{2}\right)$ at least of the second order with respect to $\lambda_{1}$ and $\lambda_{2}, \phi_{4}$ is a smooth function of $\lambda_{1}$ and $\lambda_{2}$ at least of the first order, $R$ is a $C^{\infty}$ function in variables $(x, y)$ at least of the third order with respect to $(x, y)$, and the coefficients depend smoothly on $\lambda_{1}$ and $\lambda_{2}$. Let $X=$ $x-\frac{1}{2} \phi_{2}\left(\lambda_{1}, \lambda_{2}\right), Y=y$, and denote

$\gamma_{1}=\frac{4 a_{0} x_{0}}{\mu_{0}} \lambda_{2}+\tilde{\phi}_{1}\left(\lambda_{1}, \lambda_{2}\right), \quad \gamma_{2}=\frac{\frac{x_{0} r}{2 a_{0}} \lambda_{1}-\lambda_{2}}{\sqrt{\frac{\mu_{0} r}{8 a_{0}}}}+\tilde{\phi}_{3}\left(\lambda_{1}, \lambda_{2}\right), \quad \gamma_{3}=\frac{\frac{r}{2 x_{0}}}{\sqrt{\frac{\mu_{0} r}{8 a_{0}}}}+\tilde{\phi}_{4}\left(\lambda_{1}, \lambda_{2}\right)$,

where $\tilde{\phi}_{1}, \tilde{\phi}_{3}$, and $\tilde{\phi}_{4}$ have the same properties as $\phi_{1}, \phi_{3}$, and $\phi_{4}$. Obviously, the above local parameter representation change is nonsingular. Now system (3.20) becomes

$$
\begin{aligned}
& \dot{X}=Y, \\
& \dot{Y}=\gamma_{1}+\gamma_{2} Y+X^{2}-\gamma_{3} X Y+Q\left(x, y, \lambda_{1}, \lambda_{2}\right),
\end{aligned}
$$

where $Q$ has the same properties as $R$.

By the theorems in [4] and [5], we obtain the following local representations of the bifurcation curves:

1. The saddle-node bifurcation curve $S N=\left\{\left(\gamma_{1}, \gamma_{2}\right): \gamma_{1}=0\right\}$.

2. The Hopf bifurcation curve $H=\left\{\left(\gamma_{1}, \gamma_{2}\right): \gamma_{2}=-\frac{r}{2 x_{0}} \sqrt{-\gamma_{1} \frac{\mu_{0} r}{8 a_{0}}}, \gamma_{1}<0\right\}$. 


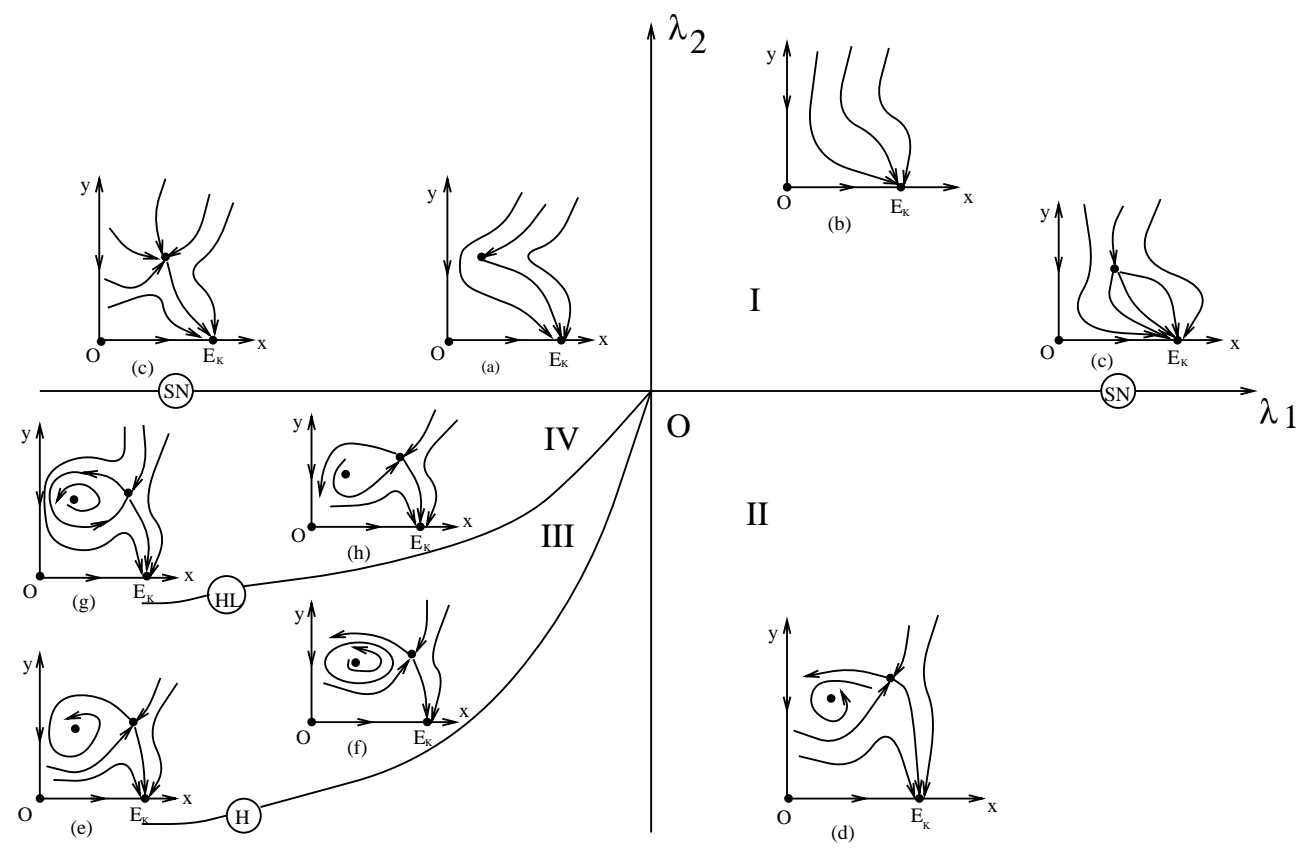

FIG. 3.1. The bifurcation set and the corresponding phase portraits of system (3.15). $E_{K}=$ $\left(K_{0}+\lambda_{1}, 0\right)$.

3. The homoclinic bifurcation curve $H L=\left\{\left(\gamma_{1}, \gamma_{2}\right): \gamma_{2}=-\frac{5 r}{14 x_{0}} \sqrt{-\gamma_{1} \frac{\mu_{0} r}{8 a_{0}}}, \gamma_{1}<\right.$ $0\}$.

We sketch these bifurcation curves in a small neighborhood of the origin in the $\left(\lambda_{1}, \lambda_{2}\right)$ plane by their first approximations and obtain the bifurcation diagram (see Figure 3.1). The bifurcation curves divide the parameter plane into four parts: I, II, III, and IV.

(a) When the parameter values are zero, system (3.15) has a unique degenerate interior equilibrium, a cusp of codimension 2.

(b) When the parameter values are in the region I, system (3.15) has no interior equilibria and every solution tends to the equilibrium $\left(K_{0}+\lambda_{1}, 0\right)$. Hence, the predator will go extinct.

(c) When the parameter values lie on the saddle-node bifurcation curve $S N$, system (3.15) has a unique degenerate interior equilibrium, a saddle node. The phase portraits can be sketched into two cases: $\lambda_{1}>0$ and $\lambda_{1}<0$.

(d) When the parameter values are in the region II, system (3.15) has two interior equilibria: one is a stable focus and the other is a hyperbolic saddle. There exists an open set of initial population densities such that both predator and prey approach to a stable steady state.

(e) When the parameter values lie on the Hopf bifurcation curve $H$, system (3.15) has two interior equilibria: one is a stable weak focus, the other is a hyperbolic saddle; and there is no periodic orbit.

(f) When the parameter values are in the region III, system (3.15) has two interior equilibria and a unique stable limit cycle. Thus there exists an open set of initial population densities such that both the predator and the prey tend to 
a stable oscillation.

(g) When the parameter values lie on the homoclinic bifurcation curve $H L$, system (3.15) has a stable homoclinic loop.

(h) When the parameter values are in the region IV, system (3.15) has two interior equilibria and no periodic orbits. From the corresponding phase portrait we can see that there exist only two initial population densities such that the predator and prey can coexist.

We have obtained all possible phase portraits for system (3.15) when the parameter values vary in a small neighborhood of the origin in the $\left(\lambda_{1}, \lambda_{2}\right)$ plane.

4. Discussion. Codimension 2 bifurcations of some standard predator-prey systems have been observed and studied in Bazykin et al. [3], Hainzl [17, 18], Xiao and Ruan [38], etc. In this paper, we have considered a predator-prey system with nonmonotonic functional response. We made a global qualitative analysis of the model depending on all parameters and showed that the system exhibits the BogdanovTakens bifurcation. By choosing the carrying capacity of the prey and the death rate of the predator as bifurcation parameters, it has been shown that the system undergoes a series of bifurcations including the saddle-node bifurcation, the supercritical and subcritical Hopf bifurcations, and the homoclinic bifurcation. Though our model is a specific case of the general model studied in Freedman and Wolkowicz [14], Mischaikow and Wolkowicz [28], and Wolkowicz [36], our analysis supports their results and also complements their results in the sense that we have not only found new types of bifurcations but also provided a detailed and global qualitative analysis for the specific case. Moreover, our analysis indicates that the dynamics of the predator-prey systems with nonmonotonic functional response can be much more complicated than we may have expected. In fact, we considered only the system with the simplified Monod-Haldane or Holling type-IV function

$$
p(x)=\frac{m x}{a+x^{2}} .
$$

If it takes the form of the original Monod-Haldane or Holling type-IV function, i.e.,

$$
p(x)=\frac{m x}{a+b x+x^{2}},
$$

then the system can have bifurcations of codimension 3 as observed in Rothe and Shafer [30]. Differing from codimension 2 bifurcations of vector fields, the problem of codimension 3 bifurcations of vector fields itself is incomplete (see Dumortier, Roussarie, and Satomayor [11] and Xiao [37]). Thus, the multiparameter bifurcation problem in predator-prey systems with nonmonotonic functional response is very interesting and challenging. We leave the codimension 3 bifurcation problem for future consideration.

Acknowledgments. We would like to thank Christian Jost for bringing the papers of Sokol and Howell [31] and Davidowicz, Gliwicz, and Gulati [10] to our attention. We are also grateful to the referees for their careful reading and constructive comments. 


\section{REFERENCES}

[1] J. F. Andrews, A mathematical model for the continuous culture of microorganisms utilizing inhibitory substrates, Biotechnol. Bioeng., 10 (1968), pp. 707-723.

[2] A. Andronov, E. A. Leontovich, I. I. Gordon, And A. G. Maier, Theory of Bifurcations of Dynamical Systems on a Plane, Israel Program for Scientific Translations, Jerusalem, Israel, 1971.

[3] A. D. Bazykin, F. S. Berezovskaya, G. A. Denisov, and Yu. A. Kuznetzov, The influence of predator saturation effect and competition among predators on predator-prey system dynamics, Ecological Model., 14 (1981), pp. 39-47.

[4] R. Bogdanov, Bifurcations of a limit cycle for a family of vector fields on the plane, Selecta Math. Soviet., 1 (1981), pp. 373-388.

[5] R. Bogdanov, Versal deformations of a singular point on the plane in the case of zero eigenvalues, Selecta Math. Soviet., 1 (1981), pp. 389-421.

[6] B. Boon ANd H. LANDelout, Kinetics of nitrite oxidation by nitrobacter winogradski, Biochem. J., 85 (1962), pp. 440-447.

[7] S.-N. Chow and J. K. Hale, Methods of Bifurcation Theory, Springer-Verlag, New York, Heidelberg, Berlin, 1982.

[8] S.-N. Chow, C. Li, ANd D. Wang, Normal Forms and Bifurcation of Planar Vector Fields, Cambridge University Press, Cambridge, UK, 1994.

[9] J. B. Collings, The effects of the functional response on the bifurcation behavior of a mite predator-prey interaction model, J. Math. Biol., 36 (1997), pp. 149-168.

[10] P. Davidowicz, Z. M. Gliwicz, And R. D. Gulati, Can Daphnia prevent a blue-green algal bloom in hypertrophic lakes? A laboratory test, Limnologica, 19 (1988), pp. 21-26.

[11] F. Dumortier, R. Roussarie, and J. Satomayor, Generic 3-parameter families of vector fields on the plane, unfolding a singularity with nilpotent linear part. The cusp case of codimension 3, Ergodic Theory Dynam. Systems, 7 (1987), pp. 375-413.

[12] V. H. EDWARDs, Influence of high substrate concentrations on microbial kinetics, Biotechnol. Bioeng., 12 (1970), pp. 679-712.

[13] H. I. Freedman, Deterministic Mathematical Models in Population Ecology, Monogr. Textbooks Pure Appl. Math. 57, Marcel Dekker, New York, 1980.

[14] H. I. Freedman and G. S. K. Wolkowicz, Predator-prey systems with group defence: The paradox of enrichment revisited, Bull. Math. Biol., 48 (1986), pp. 493-508.

[15] J. Guckenheimer, Multiple bifurcation problems of codimension two, SIAM J. Math. Anal., 15 (1984), pp. 1-49.

[16] J. Guckenheimer and P. Holmes, Nonlinear Oscillations, Dynamical Systems, and Bifurcations of Vector Fields, Appl. Math. Sci. 42, Springer-Verlag, New York, 1993.

[17] J. HAINZL, Stability and Hopf bifurcation in a predator-prey system with several parameters, SIAM J. Appl. Math., 48 (1988), pp. 170-190.

[18] J. HAINZL, Multiparameter bifurcation of a predator-prey system, SIAM J. Math. Anal., 23 (1992), pp. 150-180.

[19] J. K. Hale And A. S. Somolinos, Competition for fluctuating nutrient, J. Math. Biol., 18 (1983), pp. 255-280.

[20] C. S. Holling, The functional response of predators to prey density and its role in mimicry and population regulation, Mem. Entomolog. Soc. Can., 45 (1965), pp. 3-60.

[21] J. C. Holmes And W. M. Bethel, Modification of intermediate host behavior by parasites, Zoolog. J. Linnean Soc., 51 (1972), Suppl. 1, pp. 123-149.

[22] S.-B. Hsu, On global stability of a predator-prey system, Math. Biosci., 39 (1978), pp. 1-10.

[23] R. E. Koolj AND A. ZEGeling, Qualitative properties of two-dimensional predator-prey systems, Nonlinear Anal., 29 (1997), pp. 693-715.

[24] Y. A. Kuznetsov, Elements of Applied Bifurcation Theory, Appl. Math. Sci. 112, SpringerVerlag, New York, 1995.

[25] Y. Kuang And H. I. Freedman, Uniqueness of limit cycles in Gause-type models of predatorprey systems, Math. Biosci., 88 (1988), pp. 67-84.

[26] X. B. Lin, Exponential dichotomy and stability of long periodic solutions in predator-prey models with diffusion, in Partial Differential Equations, J. Wiener and J. K. Hale, eds., Longman Scientific and Technical, Harlow, UK, 1992, pp. 101-105.

[27] R. M. MAY, Limit cycles in predator-prey communities, Science, 177 (1972), pp. 900-902.

[28] K. Mischaikow and G. S. K. Wolkowicz, A predator-prey system involving group defense: A connection matrix approach, Nonlinear Anal., 14 (1990), pp. 955-969.

[29] M. L. Rosenzweig, Paradox of enrichment: Destabilization of exploitation ecosystems in ecological time, Science, 171 (1971), pp. 385-387. 
[30] F. Rothe AND D. S. Shafer, Multiple bifurcation in a predator-prey system with nonmonotonic predator response, Proc. Roy. Soc. Edinburgh Sect. A, 120 (1992), pp. 313-347.

[31] W. Sokol And J. A. Howell, Kinetics of phenol oxidation by washed cells, Biotechnol. Bioeng., 23 (1980), pp. 2039-2049.

[32] J. Sugie, R. Kohno, and R. Miyazaki, On a predator-prey system of Holling type, Proc. Amer. Math. Soc., 125 (1997), pp. 2041-2050.

[33] F. Takens, Forced oscillations and bifurcations, in Applications of Global Analysis I, Comm. Math. Inst. Rijksuniv. Utrecht, No. 3, 1974, pp. 1-59.

[34] R. J. TAYLOR, Predation, Chapman and Hall, New York, 1984.

[35] J. S. Tener, Muskoxen, Queen's Printer, Ottawa, Canada, 1965.

[36] G. S. K. Wolkowicz, Bifurcation analysis of a predator-prey system involving group defence, SIAM J. Appl. Math., 48 (1988), pp. 592-606.

[37] D. XIAO, Bifurcation in a class of saddle singularity of codimension 3 of a planar vector field, Sci. Sinica Ser. A, 36 (1993), pp. 252-262.

[38] D. XiaO and S. Ruan, Bogdanov-Takens bifurcations in predator-prey systems with constant rate harvesting, Fields Inst. Commun., 21 (1999), pp. 493-506.

[39] R. D. Yang And A. E. Humphrey, Dynamics and steady state studies of phenol biodegeneration in pure and mixed cultures, Biotechnol. Bioeng., 17 (1975), pp. 1211-1235.

[40] Y. R. ZHOU, Existence and uniqueness of a limit cycle for the system of equations $\dot{x}=\varphi(y)-$ $F(x), \dot{y}=-g(x)$, and the existence of exactly two limit cycles, Chinese Ann. Math., 3 (1982), pp. 89-102 (in Chinese). 